\title{
The H3K9 dimethyltransferases EHMT1/2 protect against pathological cardiac hypertrophy
}

\author{
Bernard Thienpont, ${ }^{1,2}$ Jan Magnus Aronsen, ${ }^{3,4}$ Emma Louise Robinson, ${ }^{1,5,6}$ Hanneke Okkenhaug, ${ }^{1}$ Elena Loche, ${ }^{1,5}$ Arianna Ferrini, ${ }^{1}$ \\ Patrick Brien,, ${ }^{1,6}$ Kanar Alkass,, ${ }^{7,8}$ Antonio Tomasso, ${ }^{7}$ Asmita Agrawal, ${ }^{1}$ Olaf Bergmann, ${ }^{7,9}$ Ivar Sjaastad, ${ }^{3}$ Wolf Reik, ${ }^{1,10,11}$ \\ and Hywel Llewelyn Roderick ${ }^{1,6}$

\begin{abstract}
${ }^{1}$ Epigenetics Programme, Babraham Institute, Cambridge, United Kingdom. ' Laboratory of Translational Cenetics, Vesalius Research Center, VIB and KU Leuven, Leuven, Belgium. ${ }^{3}$ Institute for Experimental Medical Research, University of Oslo and Oslo University Hospital, Oslo, Norway. ${ }^{4}$ Bjørknes College, Oslo, Norway. ${ }^{5}$ Wellcome Trust - MRC Institute of Metabolic Science, University of Cambridge, Cambridge, United Kingdom. 'Laboratory of Experimental Cardiology, Department of Cardiovascular Sciences, KU Leuven, Leuven, Belgium. ${ }^{7}$ Cell and Molecular Biology, Karolinska Institutet, Stockholm, Sweden. ${ }^{8}$ Department of Forensic Medicine, The National Board of Forensic Medicine, Stockholm, Sweden. ${ }^{9}$ DFG Research Center for Regenerative Therapies, Technische Universität Dresden, Dresden, Germany. ${ }^{10}$ Centre for Trophoblast Research, University of Cambridge, Cambridge, United Kingdom. ${ }^{11 T h e ~ W e l l c o m e ~ T r u s t ~ S a n g e r ~ I n s t i t u t e, ~ C a m b r i d g e, ~ U n i t e d ~ K i n g d o m . ~}$
\end{abstract}

\begin{abstract}
Cardiac hypertrophic growth in response to pathological cues is associated with reexpression of fetal genes and decreased cardiac function and is often a precursor to heart failure. In contrast, physiologically induced hypertrophy is adaptive, resulting in improved cardiac function. The processes that selectively induce these hypertrophic states are poorly understood. Here, we have profiled 2 repressive epigenetic marks, H3K9me2 and H3K27me3, which are involved in stable cellular differentiation, specifically in cardiomyocytes from physiologically and pathologically hypertrophied rat hearts, and correlated these marks with their associated transcriptomes. This analysis revealed the pervasive loss of euchromatic H3K9me2 as a conserved feature of pathological hypertrophy that was associated with reexpression of fetal genes. In hypertrophy, H3K9me2 was reduced following a miR-217-mediated decrease in expression of the H3K9 dimethyltransferases EHMT1 and EHMT2 (EHMT1/2). miR-217-mediated, genetic, or pharmacological inactivation of EHMT1/2 was sufficient to promote pathological hypertrophy and fetal gene reexpression, while suppression of this pathway protected against pathological hypertrophy both in vitro and in mice. Thus, we have established a conserved mechanism involving a departure of the cardiomyocyte epigenome from its adult cellular identity to a reprogrammed state that is accompanied by reexpression of fetal genes and pathological hypertrophy. These results suggest that targeting miR-217 and EHMT1/2 to prevent H3K9 methylation loss is a viable therapeutic approach for the treatment of heart disease.
\end{abstract}

\section{Introduction}

The heart is a remarkable organ that adapts to the changing hemodynamic needs of the organism throughout life by modulating its output. Acute increases in cardiovascular demand are met by an increase in cardiac contractility, whereas chronic increases associated with development, pregnancy, or sustained exercise are accommodated by cardiac growth. Despite this apparent plasticity, cardiovascular diseases are a prime health burden worldwide and are anticipated to increase even further (1). Pathologies such as chronic hypertension and aortic stenosis similarly induce cardiac growth, but while these responses are initially adaptive, they ultimately compromise cardiac output, leading to heart failure and death (2).

Since cardiomyocytes (CMs) in the adult heart are generally postmitotic $(3,4)$, cardiac growth is mediated by CM hypertrophy rather than proliferation. The defining characteristics of cardiac

Authorship note: B. Thienpont, J.M. Aronsen, and E.L. Robinson contributed equally to this work. I. Sjaastad and W. Reik contributed equally to this work.

Conflict of interest: W. Reik is a consultant and shareholder of Cambridge Epigenetix. License: This work is licensed under the Creative Commons Attribution 4.0 International License. To view a copy of this license, visit http://creativecommons.org/licensesby/4.0/. Submitted: May 2, 2016; Accepted: October 17, 2016.

Reference information: / Clin Invest. 2017;127(1):335-348. doi:10.1172/JCI88353. growth (altered contractility, changed geometry, hypertrophy) reflect changes in the phenotype of CMs, making this cell type pivotal to the hypertrophic response. However, the multicellular composition of the heart represents a potent confounder for analyzing hypertrophy, as CMs are outnumbered up to 4 -fold by non-CMs - a ratio that decreases further during pathological hypertrophy through a gain of fibroblasts and immune cells and death of CMs. Thus, analyses of heart tissue often only identify the composite of changes across its constituent cell types. To overcome this and allow the study of CM-specific responses in the intact heart, we used a powerful method involving fluorescence-assisted cell sorting to selectively isolate CM nuclei $(4,5)$.

To bring about the extensive morphological, functional, and cellular changes associated with pathological or physiological hypertrophy, a wholesale reprogramming of the CM transcriptome is required. Notably, pathological, but not physiological, hypertrophy is characterized by a reexpression of the fetal gene program, with possible deleterious consequences for cardiac function (6). For example, reexpression of the fetal myosin heavy chain MYH7 diminishes cardiac contractility, and muscle pyruvate kinase (PKM) accompanies a return to an energetically less favorable glycolytic metabolism $(7,8)$. It is, however, unclear how CMs depart from their adult state. Importantly, a role for the epigenome in the heart 
is increasingly being recognized; for example, histone 3 lysine 27 methylation is involved in heart development (9), and signalresponsive changes in histone deacetylation control gene expression in cardiac hypertrophy (10). Hypertrophy-associated changes in DNA methylation were also reported, although inactivation of the DNA methyltransferases Dnmt3a/3b did not affect the hypertrophic response (11). Despite this progress, it remains unknown whether or how the adult identity of the CM epigenome is lost during and contributes to disease. Two important repressive marks, methylation of lysines 9 and 27 of histone 3 (H3K9me2 and H3K27me3), mediate cellular memory by establishing gene programs during development that stabilize the differentiated state (12-14). Given that pathological hypertrophy in adult CMs is characterized by reexpression of fetal-associated transcripts, we hypothesized that this reprogramming arises through changes in these stable marks that determine and maintain the differentiated state.

By analyzing purified CM nuclei from hypertrophied hearts, we identified loss of $\mathrm{H} 3 \mathrm{~K} 9 \mathrm{me} 2$ as a distinguishing, conserved feature of pathological hypertrophy. Moreover, we show that the euchromatic histone methyltransferases EHMT1 and EHMT2 (also known as GLP and G9a or KMT1C and KMT1D) maintained these marks in adult myocytes but that pathological hypertrophic cues reduced EHMT1/2 expression posttranscriptionally by activating expression of microRNA 217 (miR-217). We found that a reduction in EHMT1/2 expression was necessary and sufficient to induce the expression of fetal-associated transcripts and pathological hypertrophy. Thus, we propose that the EHMT family of H3K9 dimethyltransferases "locks" adult CMs in their healthy adult state, protecting them against pathological hypertrophic remodeling.

\section{Results}

Pathological hypertrophy reduces H3K9me2 in cardiomyocytes. During development, cells become increasingly locked in their terminally differentiated state by stable epigenetic marks such as $\mathrm{H} 3 \mathrm{~K} 9 \mathrm{me} 2$ and H3K27me3. To investigate how these marks are altered in different forms of hypertrophy, we compared hearts from well-established models of pathological and physiological hypertrophy in rats: pathological hypertrophy was triggered over a 6-week period through an ascending aortic banding-induced (AB-induced) pressure overload, and physiological hypertrophy was induced through a 6-week exercise regime of increasing intensity. Both treatments resulted in a similar1.5-fold increase in the left ventricular (LV)/body weight (BW) ratio but yielded different functional and structural phenotypic outcomes. Specifically, AB-induced hypertrophy was associated with concentric cardiac growth, reexpression of the fetal gene program, expression of fibrosis-associated markers (both determined by quantitative realtime PCR [qRT-PCR]), and a moderate reduction in cardiac function (ejection fraction $[\mathrm{EF}]$, determined by echocardiography) (Figure 1, A-C, and Supplemental Figure 1, A and B; supplemental material available online with this article; doi:10.1172/JCI88353DS1). In contrast, exercise stimulated eccentric hypertrophy, without overt activation of the fetal gene program or fibrosis markers, and enhanced cardiac function (Figure 1, A-C, and Supplemental Figure 1, A and B). Pathological remodeling induced by $\mathrm{AB}$ was intentionally of a mild phenotype to allow the study of the mechanistic factors contributing to hypertrophy induction, without possible confounding sequelae associated with heart failure.
Having validated these hypertrophy models, we next profiled H3K9me2 and H3K27me3 occupancy using ChIP, followed by high-throughput sequencing (ChIP-seq), and this was done specifically in flow-sorted pericentriolar material 1-positive (PCM1+) CM nuclei ( $n=4$ for each) (Supplemental Figure 1, C and D). Consistent with studies in other tissues, these histone modifications marked broad, homogeneous segments of the genome (Supplemental Figure 1, C and E) known as broad local enrichments (BLOCs) and large, organized chromatin K9 modifications (LOCKs) (15, 16). Of note, H3K9me2 and H3K27me3 levels were substantially altered in a subset of these genome segments during hypertrophy (Figure 1D). The changes were more pronounced in pathological hypertrophy than in physiological hypertrophy, however: $9 \%$ and $0.9 \%$, respectively, of the genome showed more than $20 \%$ change in $\mathrm{H} 3 \mathrm{~K} 9 \mathrm{me} 2$, and $3.5 \%$ and $0.9 \%$ showed more than $20 \%$ change in H3K27me3 (FDR = 10\%; Figure 1D). Remarkably, hypertrophy induced by $\mathrm{AB}$ was almost exclusively associated with a loss of H3K9me2. This pervasive loss was also evident by immunofluorescence of heart sections and by immunoblotting, which revealed a decrease of $40 \%$ to $60 \%$ in $\mathrm{H} 3 \mathrm{~K} 9 \mathrm{me} 2$ levels in CM nuclei upon $\mathrm{AB}$ (Figure 1E and Supplemental Figure 1F), whereas H3K9me2 levels remained unchanged with exercise (Figure 1E and Supplemental Figure 1G). Likewise, consistent with the lower number of BLOCs exhibiting alterations in $\mathrm{H} 3 \mathrm{~K} 27 \mathrm{me} 3$, global levels of H3K27me3 were unaffected by either form of hypertrophy at this time point (Supplemental Figure 1, H and I). Changes at other time points and of other histone modifications cannot be excluded.

Pathological hypertrophy reduces $\mathrm{H} 3 \mathrm{~K} 9 \mathrm{me} 2$ at fetal heart genes. We next characterized the regions of the genome affected by loss of H3K9me2. A systematic ontology term analysis of genes in regions that were decreased in $\mathrm{H} 3 \mathrm{~K} 9 \mathrm{me} 2$ upon $\mathrm{AB}$ revealed an enrichment of hypertrophy-associated processes including signaling, responses to hormone stimuli, and heart development (Figure 2A). Genes depleted in H3K9me2 included the fetal gene program canonical markers atrial natriuretic peptide (Nppa), brain natriuretic peptide $(N p p b)$, and $M y h 7$ (Figure 1D and Figure 2B, and Supplemental Figure 1J). These marks thus appear to repress the fetal gene program in the adult $\mathrm{CM}$. To establish that $\mathrm{H} 3 \mathrm{~K} 9 \mathrm{me} 2 \mathrm{is}$ indeed acquired during CM maturation, we profiled a time series of postnatal CM nuclei (P1 to adult) for H3K9me2 using ChIP-seq, which revealed a widespread gain in $\mathrm{H} 3 \mathrm{~K} 9 \mathrm{me} 2$ during $\mathrm{CM}$ maturation that was particularly evident for LOCKs that showed a loss of H3K9me2 upon AB (Figure 2C), including Nppa, Nppb, and Myh7 (Supplemental Figure 1, K and L). Immunofluorescence analyses again underlined the pervasive nature of this H3K9me2 acquisition (Figure 2D). In conclusion, we observed a widespread and substantial loss of $\mathrm{H} 3 \mathrm{~K} 9 \mathrm{me} 2$ upon hypertrophy induced by $\mathrm{AB}$ but not by exercise. Our data suggest that pathological hypertrophic cues strip CMs of this epigenetic constraint imposed during development, thereby enabling a reactivation of the fetal gene program and a pathological remodeling of gene expression.

Loss of H3K9me2 correlates with increased transcription in hypertrophic cardiomyocytes. To assess whether the epigenetic changes observed underlie transcriptional regulation, we next used RNAseq to profile transcriptomes of sorted CM nuclei from hearts subjected to physiological and pathological hypertrophy. In addition to providing a CM-specific transcriptome, this nuclear RNA also pro- 
A

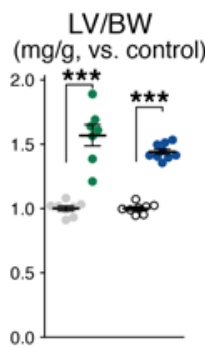

B

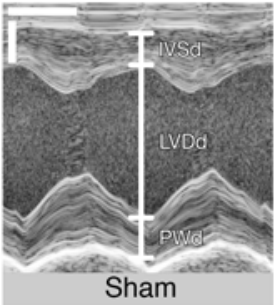

Sham

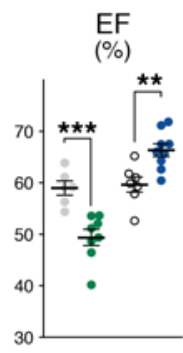

(\%)

**
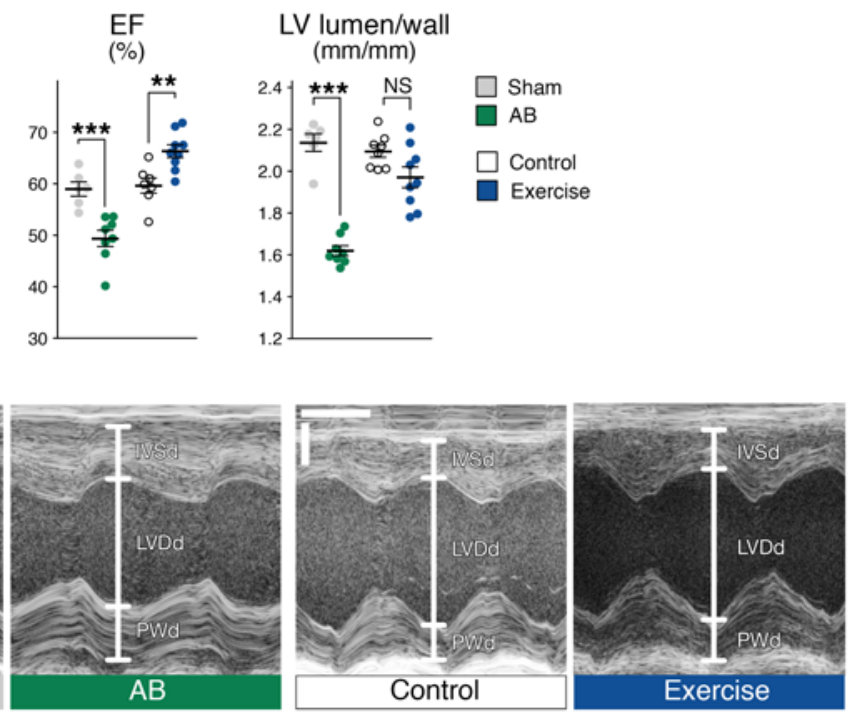

C

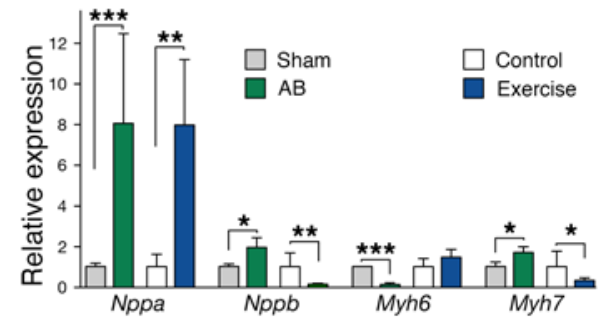

D

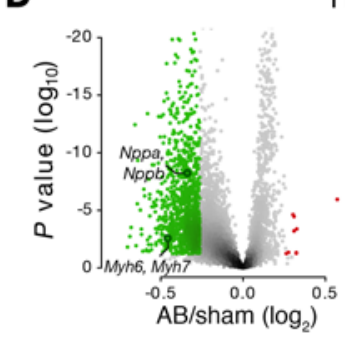

H3K9me2
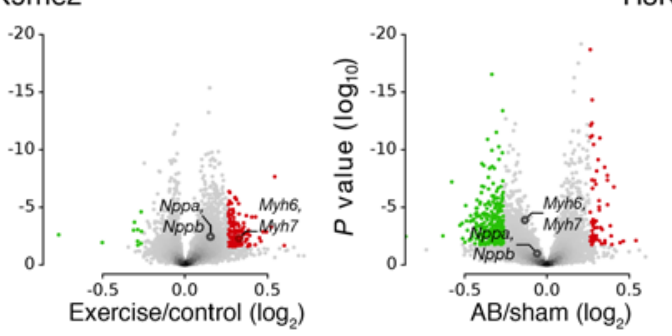

H3K27me3

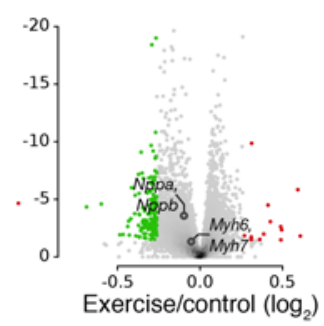

E

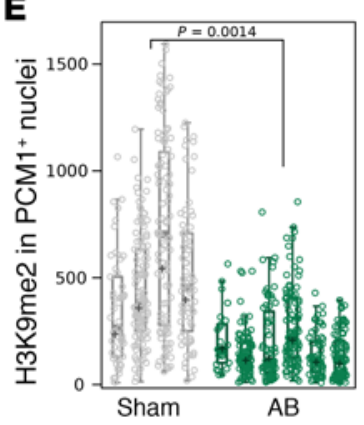

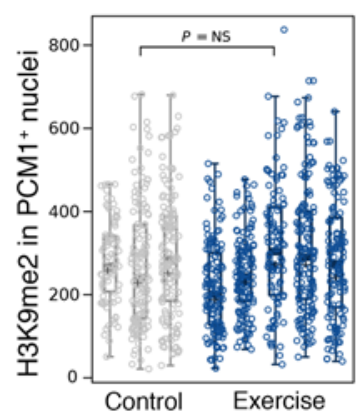
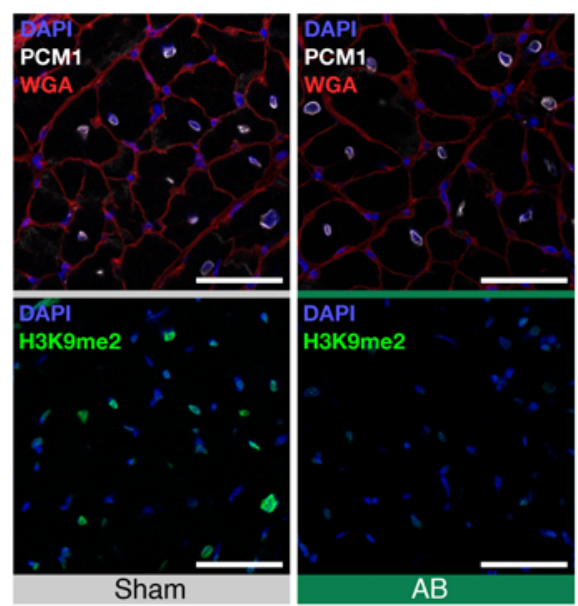

Figure 1. H3K9me2 is decreased in CMs upon AB in rats. (A) Graphs show LV/BW ratio, normalized to sham or control (mg/g); EF percentage; and ratio of LV lumen diameter to myocardial wall thickness at end-diastole. $n=7,9,6$, and 8 animals for control, exercise, sham, and AB, respectively. (B) M-mode echocardiogram showing, at end-diastole, the LV diameter (LVDd) and thickness of the posterior wall (PWd) and of the interventricular septum (IVSd). Horizontal scale bars: 0.1 second; vertical scale bars: $2 \mathrm{~mm}$. (C) qRT-PCR showing expression of Nppa, Nppb, Myh6, and Myh7 in the LV. (D) Volcano plot of differential markings by H3K9me2 and H3K27me3 of, respectively, LOCKs and BLOCs in PCM1+ nuclei upon pathological (left) and physiological (right) hypertrophy, as determined by ChIP-seq. Red and green dots represent regions enriched and depleted upon hypertrophy (FDR $<10 \% ;>20 \%$ change). (E) Plots show H3K9me2 signal in PCM1+ nuclei, determined by immunofluorescence analysis of the LV from AB, sham-operated, exercise, and control animals ( $n=4,5,3$, and 5, respectively). Representative confocal immunofluorescence images show staining for wheat germ agglutinin (WCA, red) and PCM1 (white), and H3K9me2 (green) in the LV. Scale bars: $50 \mu \mathrm{m}$. Error bars indicate the SEM. $n=5$ (C) and 4 (D) replicates. ${ }^{*} P<0.05,{ }^{* *} P<0.01$, and ${ }^{* *} P<0.001$, by Student's $t$ test (A and C) and nested ANOVA (E).

vided the benefit of establishing levels of active transcription at the point of heart removal. Of note, our sorting protocol did not affect the nuclear transcriptome, as a comparison between samples before and after sorting revealed expression of the same genes as well as highly similar intronic RNA transcript counts (Pearson's $r^{2}=0.91$ ), although, as expected, nuclear RNA was depleted of mitochondrial transcribed RNAs (Supplemental Figure 2, A-C). In addition, RNA from PCM1+ nuclei was highly enriched in marker genes and 
A

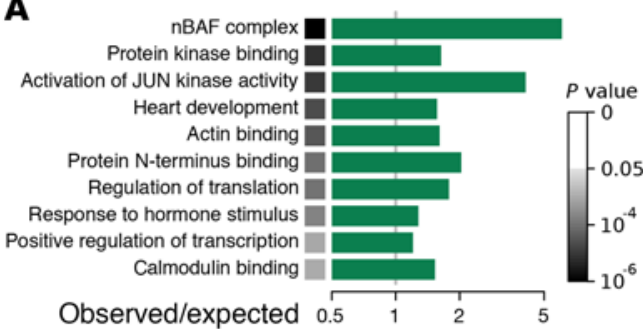

B

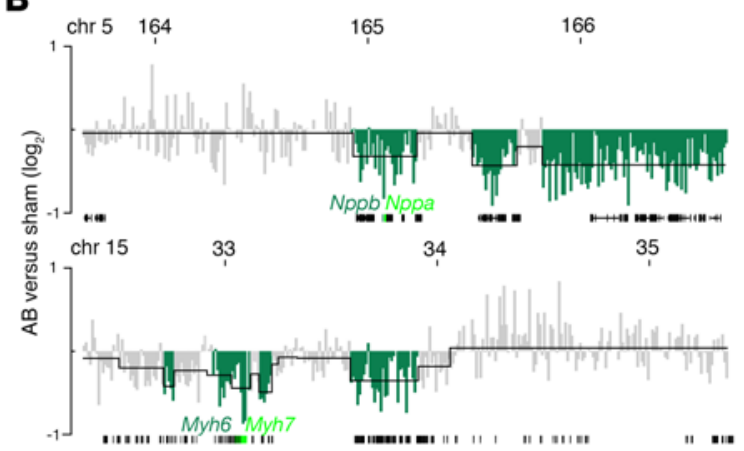

C
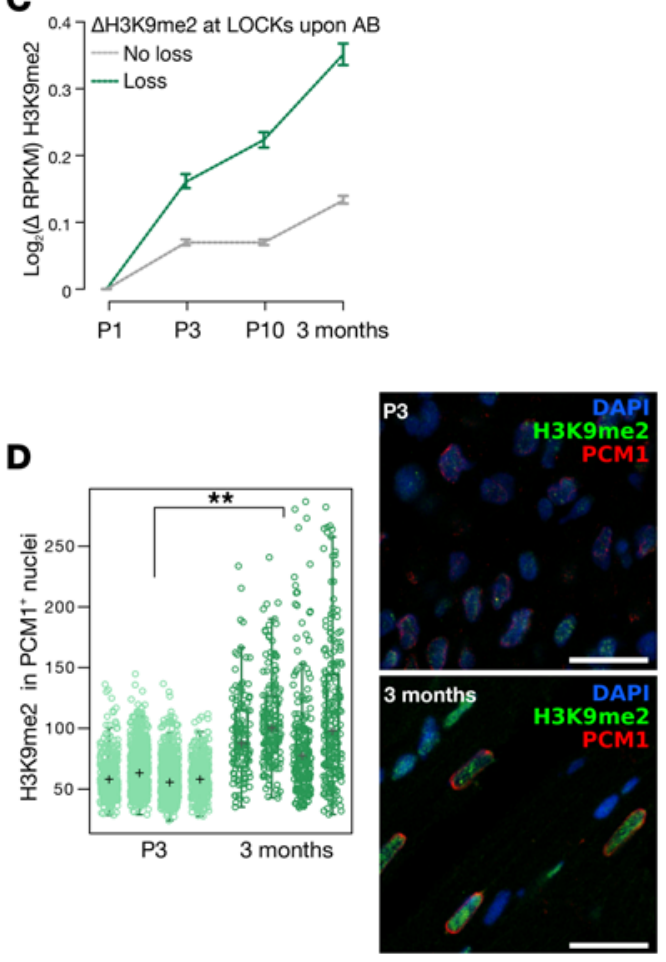

Figure 2. H3K9me2 is acquired during cardiomyocyte maturation and lost at fetal genes upon $\mathbf{A B}$. (A) Selected ontology terms enriched among genes in regions altered in $\mathrm{H} 3 \mathrm{~K} 9 \mathrm{me} 2$ upon $\mathrm{AB}$. (B) H3K9me2 enrichment at loci on chromosomes (Chr) 5 and 15 in PCM1+ nuclei. Enrichment is displayed per $10-k b$ bin as $\log _{2}$ (ratio of $A B$ vs. sham). Green denotes significantly altered LOCKs. (C) Changes in H3K9me2 during CM maturation at LOCKs showed loss or no loss (green or gray) upon AB. (D) Quantification and representative images of immunofluorescence staining of H3K9me2 (green) in CMs (PCM1, red) in neonatal (3-day-old) and adult (3-month-old) mice ( $n=4$ each). Hypertrophy was induced for 6 weeks. Error bars indicate the SEM. $n=4$ (B and $\mathbf{D})$ and 2 (C) replicates. ${ }^{* *} P<0.01$, by nested ANOVA (D). processes specific to $\mathrm{CMs}$, whereas $\mathrm{PCM1}^{-}$nuclei were enriched in markers of other cardiac cell types (fibroblasts and endothelial and immune cells), highlighting the purity (>98\%) and proper identity of our CM nuclei (Supplemental Figure 2, D-G). Overlaying epigenomic and transcriptomic profiles revealed that genes and regions heavily covered by $\mathrm{H} 3 \mathrm{~K} 9$ me 2 or $\mathrm{H} 3 \mathrm{~K} 27$ me 3 were transcriptionally silent, as anticipated (Figure 3, A and B, Supplemental Figure 2, H and I) (17). Notably, however, whereas H3K9me2 levels decreased linearly with increasing levels of transcription, H3K27me3 levels were consistently low across the expressed genes (Figure 3, A and $\mathrm{B}$ and Supplemental Figure 2, J and K). This suggests that, in adult CMs, H3K9me2 is involved in modulating gene expression levels, while H3K27me3 marks an on/off state.

In line with the more marked epigenetic changes observed upon $\mathrm{AB}$, we also noted that transcriptomic changes were more pronounced in pathological hypertrophy than in physiological hypertrophy (Figure 3, C and D), with 705 and 331 proteincoding genes $(3.1 \%$ and $1.4 \%)$, respectively, being differentially expressed (FDR 10\%; Supplemental Tables 1 and 2). A comparison between differentially expressed genes here and those found in studies in which transcriptional changes were assessed in heart tissues confirmed that genes overexpressed in non-CMs were overrepresented in tissue-based profiles but not in our pure CM profiles, further validating our sorting approach (Supplemental Figure 2L). Genes that increased in expression upon AB included established pathological hypertrophy marker genes such as Nppa, $N p p b$, and $M y h 7$ (Figure 3C). These genes were also depleted in H3K9me2 (Figure 1D and Figure 2B). A global comparison of changes in $\mathrm{H} 3 \mathrm{~K} 9 \mathrm{me} 2$ and gene expression revealed that regions that were decreased in $\mathrm{H} 3 \mathrm{~K} 9 \mathrm{me} 2$ upon $\mathrm{AB}$ were highly enriched in genes that were upregulated in response to this intervention (Figure $3 \mathrm{E}$ and Supplemental Figure 2M), suggesting that H3K9me2 alterations underlie gene expression changes. Notably, the few loci that exhibited gains in $\mathrm{H} 3 \mathrm{~K} 9 \mathrm{me} 2$ levels upon $\mathrm{AB}$ (Figure 1D) were associated with 61 genes that were either not expressed in the heart (including genes involved in olfaction) or expressed at very low levels. Ontological analysis of genes upregulated upon $\mathrm{AB}$ revealed that they encode proteins involved in a diverse array of processes including heart contraction, cardiac morphogenesis, and signaling, processes that were similarly enriched among genes showing an $\mathrm{AB}$-associated loss of H3K9me2 (Figure $3 \mathrm{~F}$ ). To further determine whether genes that lose $\mathrm{H} 3 \mathrm{~K} 9 \mathrm{me} 2$ upon $\mathrm{AB}$ are part of the wider transcriptional program of the fetal heart, we also assessed their expression during in vitro differentiation of embryonic stem cells (ESCs) into CMs (18) and during maturation of the postnatal heart (19). This assessment revealed that genes losing $\mathrm{H} 3 \mathrm{~K} 9 \mathrm{me} 2$ upon $\mathrm{AB}$ were strongly enriched among genes that were upregulated during in vitro differentiation into CMs, as well as among genes downregulated in the heart after birth $\left(P<10^{-16}\right.$ for both; Figure 3, G and $\left.\mathrm{H}\right)$, which was in line with the observed gain of $\mathrm{H} 3 \mathrm{~K} 9 \mathrm{me} 2$ upon $\mathrm{CM}$ maturation (Figure 2, C and D). Together, these data show that loss of H3K9me2 correlates with an overexpression of hypertrophy-induced genes, including the fetal gene program. Such transcriptional changes, although compensatory at the onset, are considered to reduce the efficiency of CM contraction and metabolism and thus to underlie pathological cardiac remodeling (6). 
A

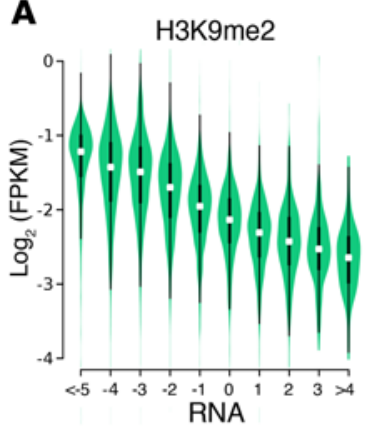

RNA
B

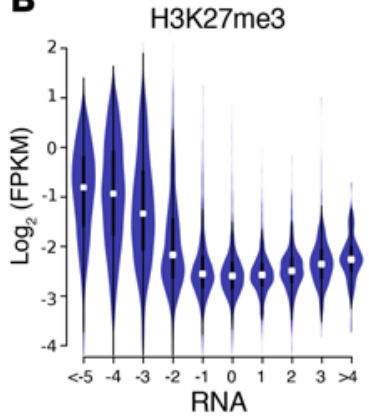

C

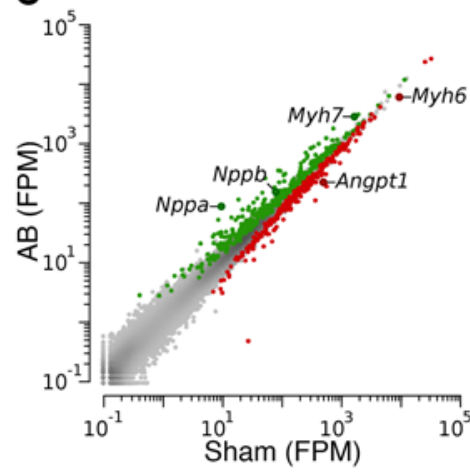

D

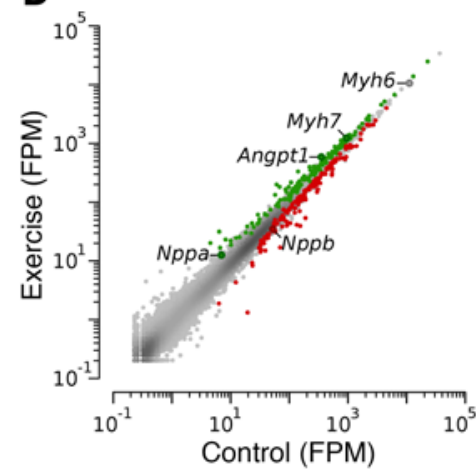

E

Differentially expressed genes, stratified by $\Delta \mathrm{H} 3 \mathrm{~K} 9 \mathrm{me} 2$

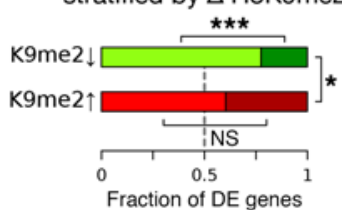

$\square$ Increased expression

$\square$ Decreased expression
$\mathbf{F}$

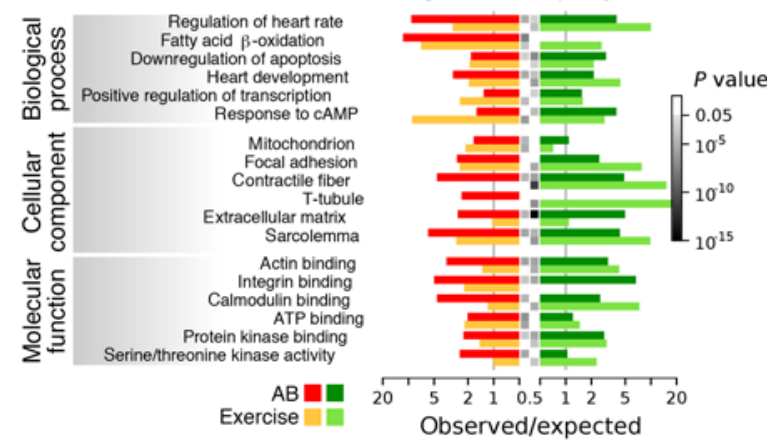

G

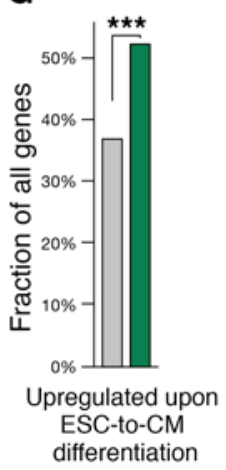

H

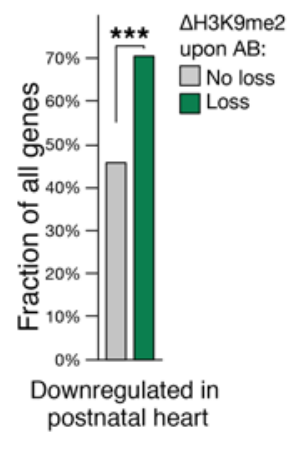

Figure 3. Hypertrophy-associated transcriptomic changes in flow-sorted rat CMs. (A and B) Violin plots illustrating the gene-wise relation between RNA expression and H3K9me2 (A) or H3K27me3 (B). Data were quantified per gene and are represented as log ((fragments +1$)$ per kb per million) (FPKM). (C and D) Gene expression, represented as fragments per million (FPM), in PCM1+ nuclei sorted from hypertrophied and control hearts. Highlighted are the genes that were significantly upregulated (green) and downregulated (red) (FDR $<10 \%$ ) after 6 weeks of hypertrophy induction, with characteristic genes indicated. (E) Fractions of differentially expressed genes (FDR $<10 \%$, difference $>20 \%$ ) in LOCKs with H3K9me2 loss or gain upon AB. (F) Selected ontological terms significantly enriched among genes significantly up- or downregulated in $\mathrm{PCM} 1^{+}$nuclei upon exercise and $\mathrm{AB}$. The gray box before each bar represents the $P$ value of enrichment; there are no boxes for $P$ values greater than 0.05 . (G and $\mathbf{H}$ ) Characterization of genes displaying loss of H3K9me2 upon AB. (G) Graph represents the fraction of genes that were upregulated during the in vitro differentiation of ESCs into CMs, as determined by Wamstad and colleagues (18). (H) Graph shows the fraction of genes that were downregulated in the adult versus newborn heart (19). Data for $\mathbf{G}$ and $\mathbf{H}$ were from the mouse genome. For comparisons with the rat genome, analyses were limited to genes having 1 -to-1 paralogs between both species. $n=4$. ${ }^{*} P<0.05$ and ${ }^{* * *} P<0.001$, by $\chi^{2}$ test.

Pathological hypertrophy reduces EHMT1/2 expression. EHMT1 and EHMT2 are obligate heterodimeric partners that catalyze H3K9 dimethylation, predominantly at euchromatic loci at the nuclear periphery (20-23). Our ChIP-seq analysis demonstrated that upon $\mathrm{AB}$, the majority of $\mathrm{H} 3 \mathrm{~K} 9 \mathrm{me} 2$ was lost at expressed, euchromatic loci. Furthermore, immunofluorescence in $A B$ hearts showed that $\mathrm{H} 3 \mathrm{~K} 9 \mathrm{me} 2$ was predominantly lost at the $\mathrm{CM}$ nuclear periphery (Supplemental Figure 3A). We therefore assessed the expression of Ehmt1/2 in sorted CMs. A profound decrease in RNA expression $(-60 \%)$ of both enzymes was noted upon pathological, but not physiological, hypertrophy (Figure 4, A and C). Immunofluorescence and immunoblot analyses confirmed the loss of EHMT1/2 proteins upon pathological hypertrophy in CMs (Figure 4, B and D, and Supplemental Figure 3B). This substantial reduction in EHMT1/2 expression was sufficient to explain the loss of H3K9me2, precluding a requirement to invoke a major contribution from an altered targeting specificity of EHMT1/2.

We similarly assessed the involvement of H3K9 demethylases and other methyl transferases by gene expression analyses (Supplemental Figure 3, C and D). However, while expression of the H3K9me3 demethylases Kdm $4 a$ and $K d m 4 b$ was increased upon
$\mathrm{AB}$, no H3K9me2 demethylases or other methyltransferases were altered $(24,25)$. Together, these findings implicate a loss of EHMT1 and EHMT2 in the AB-induced loss of H3K9me2, and not a loss of other methyltransferases or gain of $\mathrm{H} 3 \mathrm{~K} 9 \mathrm{me} 2$ demethylases.

EHMTs protect cardiomyocytes from pathological hypertrophy in vitro. We next determined whether the observed reduction in EHMT1/2 activity following AB is causal for the induction of pathological hypertrophy by modulating EHMT1/2 activity and abundance. In primary cultures of neonatal rat ventricular myocytes (NRVMs), pathological hypertrophy can be modeled by stimulation of Gq signaling pathways through endothelin 1 (ET-1) application (26-28). As observed upon pathological hypertrophy in vivo, exposure to ET-1 $(100 \mathrm{nM})$ resulted in the expression of established markers of CM hypertrophy. Specifically, ET-1 induced mRNA expression of the fetal gene program ( $N p p a, N p p b$, and $M y h 7$, but not of the adult isoform $M y h 6$ ), increased the abundance of atrial natriuretic factor (ANF, the product of the Nppa gene), increased sarcomere organization, and increased protein synthesis (Figure 5, A-D). Importantly, as observed during pathological hypertrophy in vivo, ET-1 application reduced Ehmt1 and Ehmt 2 expression and H3K9me2 levels (Supplemental Figure 4, A and B). Next, we inac- 
A
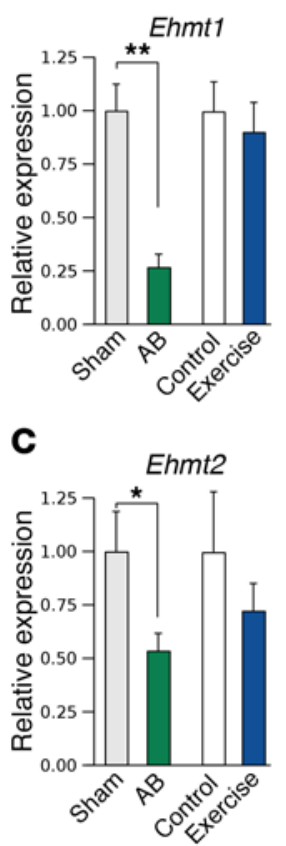

B
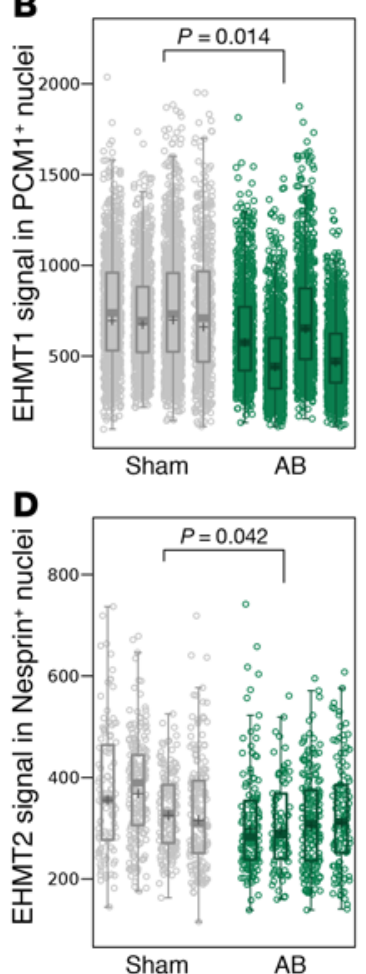
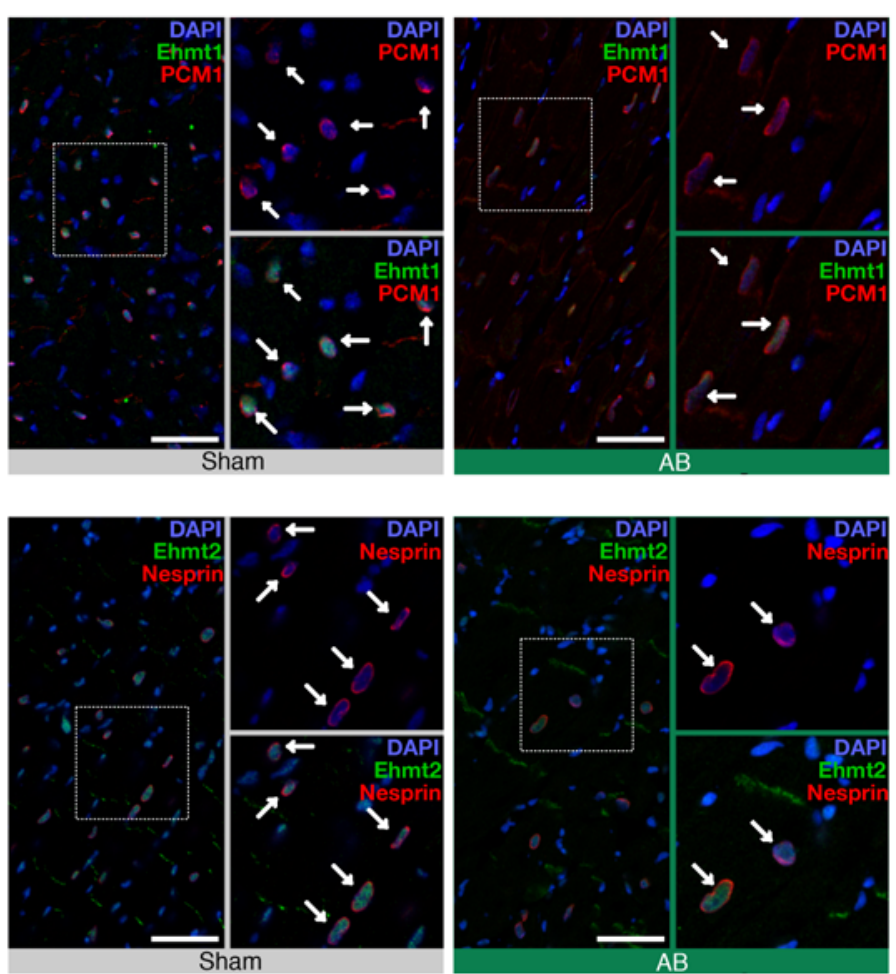

Figure 4. EHMT1/2 expression is decreased following AB in rats. (A and $\mathbf{C})$ qRT-PCR of Ehmt1 (A) and Ehmt2 (C) mRNA expression in sorted PCM1+ nuclei isolated from rats subjected to AB or exercise for 6 weeks. Error bars represent the mean \pm SEM of 4 (sham, AB) or 7 (control, exercise) biological replicates. ${ }^{*} P<0.05$ and ${ }^{* *} P<0.01$, by Student's $t$ test. (B and $\mathbf{D}$ ) (left) jitter plot illustrating EHMT1 and EHMT2 expression ( $n=4$ for all conditions) in PCM1 ${ }^{+}$and nesprin ${ }^{+}$cardiomyocytes from hearts as in A. $P$ values were calculated using nested ANOVA. Representative images of immunofluorescence staining for PCM1 (red) and EHMT1 (green) (B) and nesprin (red) and EHMT2 (green) (D) in LV sections from rats that underwent sham operation or AB. The dashed white box indicates the location of the zoomed images (zoom, $\times 2$ ) to the right of each panel. Scale bars: $50 \mu \mathrm{m}$. Nuclei were counterstained with DAPI (blue), and CM nuclei are indicated by arrows.

tivated EHMTs using a recently developed small molecule, A-366, that has potent and specific inhibitory effects on EHMT1 and EHMT2 activity in biochemical assays and cultured cells $(29,30)$. A-366 $(0.5 \mu \mathrm{M})$ decreased H3K9me2 levels in NRVMs, without cytotoxicity (Supplemental Figure 4, C and D). Importantly, A-366 application alone was sufficient to induce hypertrophy: it increased protein synthesis, ANF protein expression, sarcomere organization, as well as Nppa, Nppb, and Myh7, but not Myh6, mRNA expression (Figure 5, A-D). This indicates that EHMT1/2 inhibition phenocopies the induction of pathological hypertrophy by ET-1. Indeed, in contrast, physiological hypertrophy, as induced by 10 nM insulin-like growth factor 1 application (IGF-1) (31), downregulated Nppb, upregulated Myh6, and did not affect Myh7, Ehmt1, or Ehmt2 expression (Supplemental Figure 4E). The effect of ET-1 and A-366 upon hypertrophy marker expression was generally not summative. Together with the observed reduction of Ehmt expression upon ET-1 treatment, this indicated that decreased EHMT1/2 activity is downstream of ET-1. A similar pro-hypertrophic effect was observed using a second EHMT1/2 inhibitor, UNC0366 (350 $\mathrm{nM}$ ), which is structurally dissimilar to A-366 (Supplemental Figure $4, \mathrm{~F}$ and G) $(29,30)$. Sufficiency of A-366 or UNC0638 to elicit a pathological hypertrophic response in NRVMs strongly suggested specificity of the effects observed. Finally, with the aim of rescuing the effect of a reduction in EHMTs during pathological hypertro- phy, we used adenovirus to overexpress EHMT2 in NRVMs (Supplemental Figure 4H). EHMT2 overexpression was sufficient to abrogate the ET-1-induced loss of H3K9me2 and the associated hypertrophic response (Figure 5, E and F, and Supplemental Figure 4I), demonstrating that loss of EHMT activity is not only sufficient but also necessary to induce a pathological hypertrophic response.

EHMT1/2 inhibition induces pathological cardiac hypertrophy. Prompted by our in vitro findings, we next investigated the impact of EHMT1/2 inhibition on cardiac hypertrophy in vivo in 2 murine models. In a pharmacological model, mice were implanted with osmotic minipumps delivering either carrier or A-366 (30 mg/kg BW/day), whereas in a genetic model, the Ehmt2 locus was specifically and conditionally inactivated in CMs of the adult mouse heart (Supplemental Figure 5, A-C). Confocal imaging of immunostained tissue sections confirmed a reduction in $\mathrm{CM}$ H3K9me 2 levels by both interventions (Figure 6, A and B). The reduction in $\mathrm{H} 3 \mathrm{~K} 9 \mathrm{me} 2$ levels was comparable between mice with Ehmt2 loss and those that received A-366, indicating a similar efficiency of both methods for EHMT inactivation (Figure 6, A and B). Importantly, EHMT inactivation in sham-operated controls induced spontaneous hypertrophy. In vivo analysis showed a time-dependent increase in LV wall thickness and a decrease in the lumen/wall thickness ratio and of the $\mathrm{EF}$, while a postmortem examination revealed an increase in the 

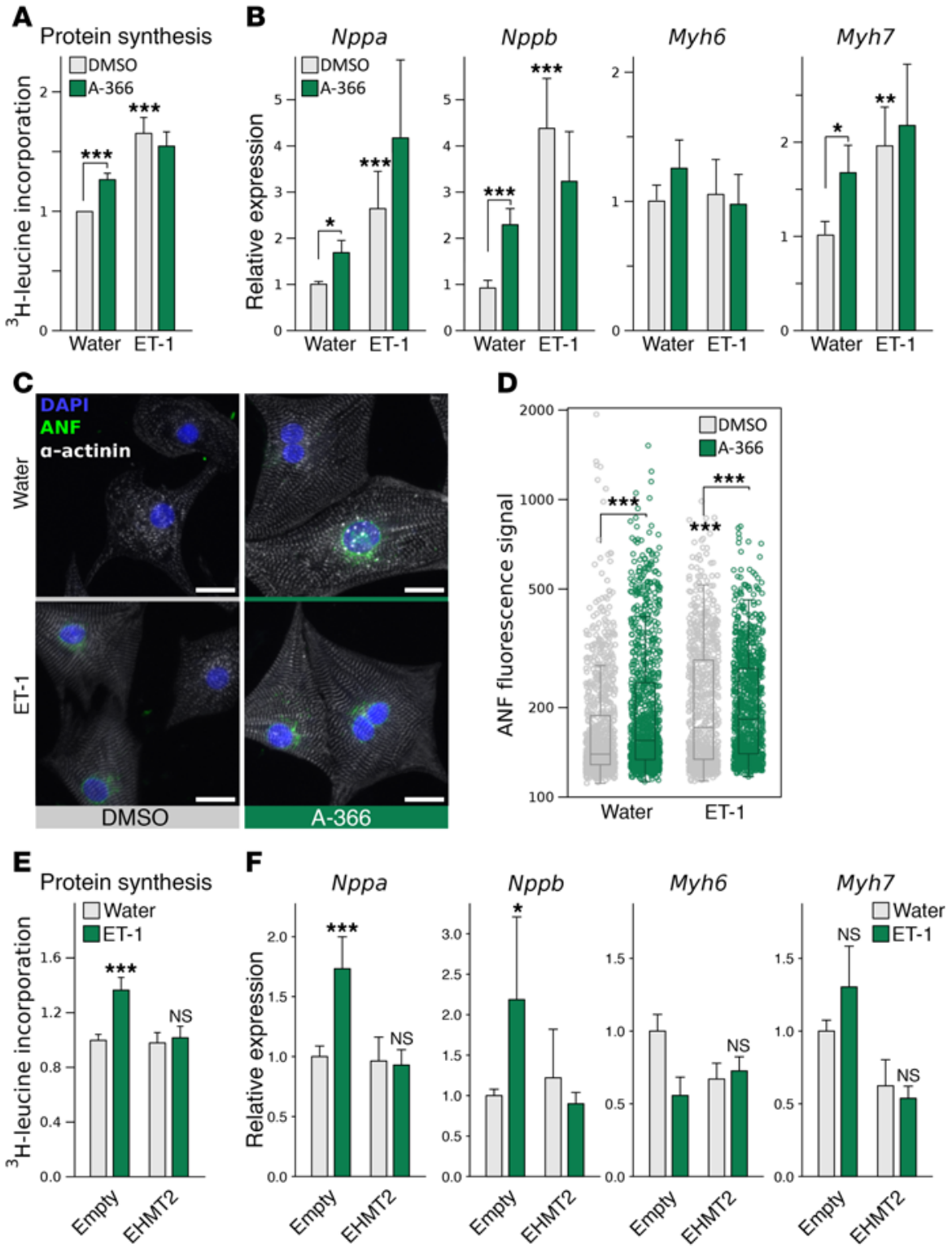

Figure 5. Suppression of EHMTs induces hypertrophy in vitro. (A and B) Protein synthesis (A) and qRT-PCR for Nppa, Nppb, $M y h 6$, and Myh7 (B) in primary NRVMs exposed for 48 hours to ET-1 or vehicle (water) and in response to EHMT1/2 inhibition (A-366). (C) Immunofluorescence staining for $\alpha$-actinin (white) and ANF (green) in NRVMs treated with or without ET-1 and/ or A-366. Nuclei were counterstained with DAPI (blue). Scale bars: $20 \mu \mathrm{m}$. (D) Immunofluorescence signal intensities of perinuclear ANF (shown is 1 representative experiment from 4 repeats). ( $E$ and $\mathbf{F}$ ) Protein synthesis analysis by ${ }^{3} \mathrm{H}$-leucine incorporation (E) and Nppa, Nppb, Myh6, and Myh7 expression (F) in NRVMs transduced with empty or EHMT2 adenovirus 8 hours prior to agonist addition (ET-1 or water). Cells were assayed 48 hours after agonist addition. Note that the hypertrophic response appears dampened in transduced NRVMs compared with nontransduced NRVMs in $\mathbf{A}$ and $\mathbf{B}$. Error bars in $\mathbf{A}, \mathbf{B}, \mathbf{E}$, and $\mathbf{F}$ represent the mean $\pm \mathrm{SEM}$. $n=5$ biological replicates. ${ }^{*} P<0.05$,

${ }^{* *} P<0.01$, and ${ }^{* *} P<0.001$, by Student's $t$ test.
$\mathrm{LV} / \mathrm{BW}$ ratio. Together, these parameters indicated a concentric, pathological form of hypertrophy that phenocopied $\mathrm{AB}$ (Figure 6, C-E, and Supplemental Figure 5, D-H). The degree of hypertrophy paralleled that of established potent hypertrophic agents such as isoproterenol and angiotensin II (32) and occurred without blood pressure alterations, indicating a primary hypertrophy that was not caused by increased afterload (Supplemental Figure 5, I and J). Last, as observed in NRVMs, EHMT inactivation induced the classical markers for maladaptive hypertrophy (including the fetal gene program), as well as fibrosis, and this occurred independently of genotype, Cre recombinase activation, or tamoxifen injection alone (Figure 6, F and G, and Supplemental Figure 5, K and L). Also, a wider set of genes from the fetal gene program that were increased upon $\mathrm{AB}$ in rat $\mathrm{CMs}$, were induced upon CM-specific Ehmt2 KO (Supplemental Figure $5 \mathrm{M}$ ). Moreover, genes induced by AB and Ehmt2 KO substantially overlapped with those with increased expression in in vitro CM-differentiated ESCs and those downregulated in in vivo cardiac maturation. These genes also showed appropriate loss in H3K9me2 upon AB (Supplemental Figure 5M).

To assess the impact of EHMT1/2 inactivation on pressure overload-induced cardiac remodeling, we performed, in parallel to the sham operations, a banding of the ascending aorta. As observed in rats (Figure 4), banding reduced Ehmt1 and Ehmt2 expression (Supplemental Figure 5N). In addition, H3K9me2 levels were reduced following banding, although not to the same extent as that observed upon exposure to A-366 or inactivation of Ehmt2 alone (Figure 6, A and B). In banded mice, A-366 administration or Ehmt2 inactivation did not exacerbate the hypertrophic response; there were no or only minor additional increases in LV weight and no further decrease in the LV wall/lumen ratio or in EF (Figure 6D and Supplemental Figure $5, \mathrm{D}-\mathrm{F})$. In performing these experiments, we noted a slightly greater $\mathrm{AB}$-induced hypertrophy in Ehmt $t^{f l f l}$ mice compared with mice infused with A-366 or with carrier (Figure 6D). The experiments involving A-366 and Ehmt genetic deletion were not 
A

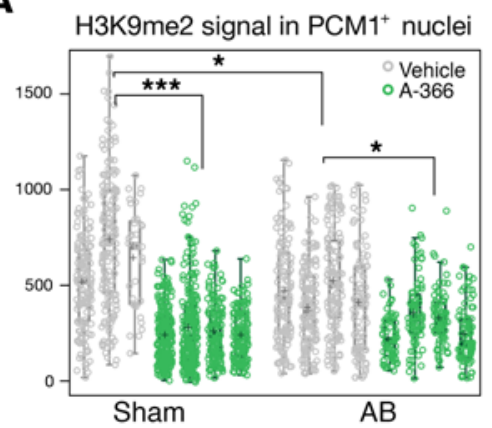

B

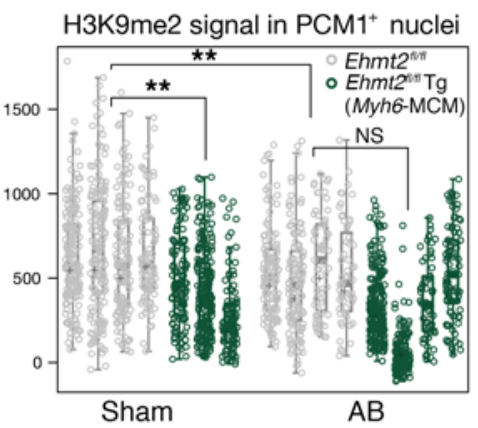

E

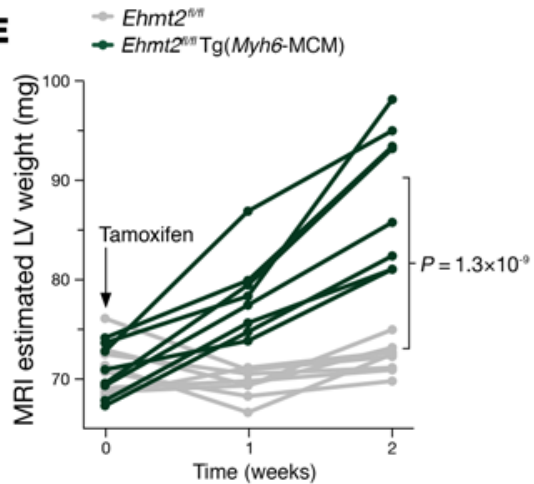

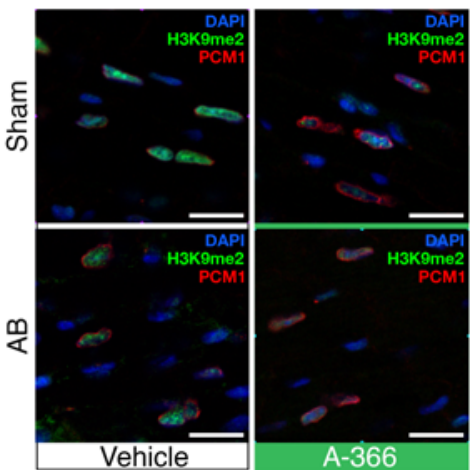

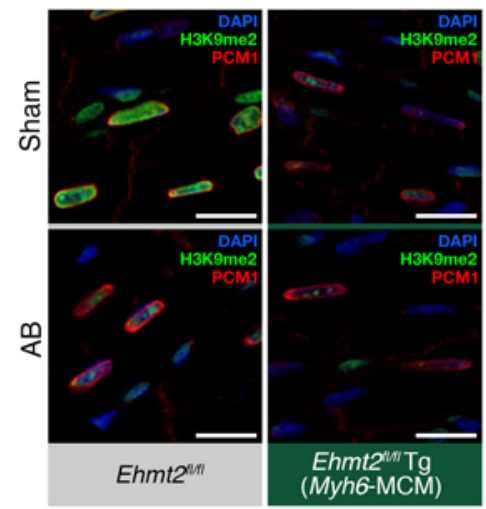

$\mathbf{F}$

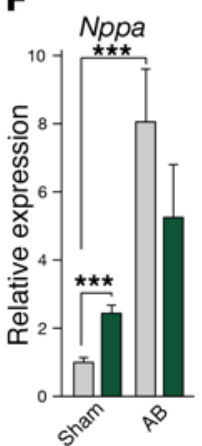

C

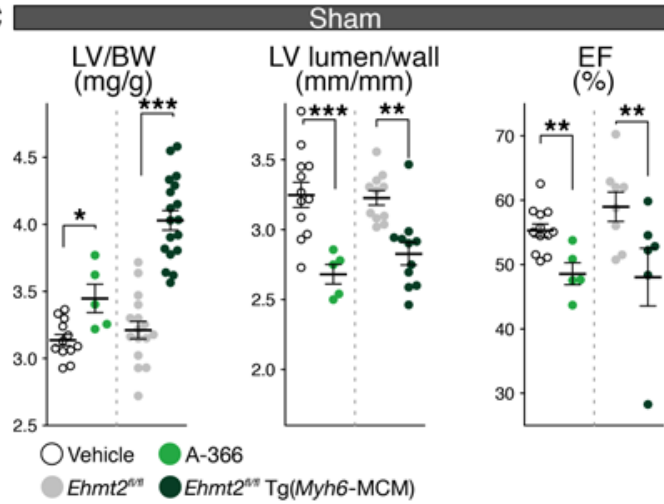

D

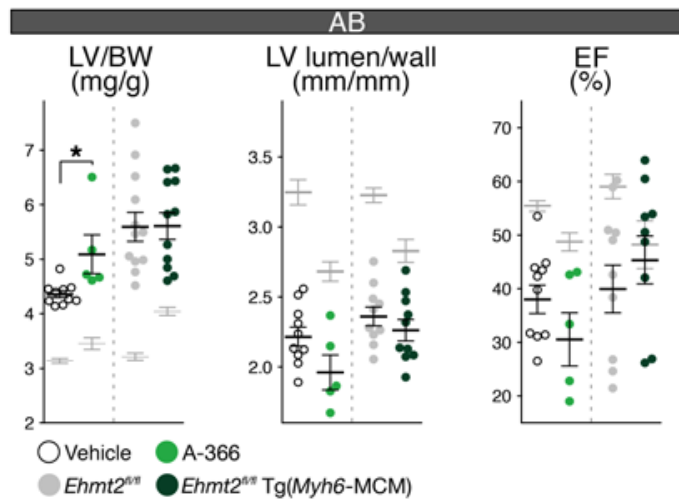

Figure 6. Pharmacological or genetic inactivation of EHMTs induces pathological hypertrophy. (A and B) H3K9me2 signal in PCM1+ nuclei and representative immunofluorescence images for PCM1 (red) and H3K9me2 (green) in sections from LVs of sham- and AB-operated mice (A) after 10 days with A-366 or vehicle administration through s.c. mini-osmotic pumps ( $n=3$ for sham vehicle and 4 for other treatments) and (B) from Ehmt $2^{f / / f l}$ and $E h m t 2^{f / / f l} \mathrm{Tg}(\mathrm{Myh} 6-\mathrm{MCM})$ mice $\left[n=3\right.$ for sham-treated Ehmt ${ }^{f / / f f} \operatorname{Tg}($ Myh6-MCM) mice and $n=4$ for all other conditions], 2 weeks after tamoxifen injection. Scale bars: $20 \mu \mathrm{m}$. Nuclei are stained with DAPI (blue). (C and D) Alterations in LV weight, shape, and EF for sham-operated mice (C) $(n=12,5,11$, and 11 mice for the indicated conditions) and $A B$-operated mice (D) $\left(n=10,5,10\right.$, and 10 mice for the indicated conditions), after 10 days of administration of A-366 or vehicle and for Ehmt $2^{f / / f l}$ and Ehmt2 $2^{f / f l} \mathrm{Tg}($ Myh6-MCM) mice, 2 weeks after tamoxifen injection. Vertical dashed line indicates experiments conducted separately to inactivate EHMT1/2 by chemical or genetic means. To enable comparison within experiments, averaged data for sham-operated animals from $\mathbf{C}$ were added to the data in $\mathbf{D}$ (grayed averages \pm SEM). (E) Evolution of LV weight following tamoxifen injection into mice of the indicated genotypes, as estimated by MRI at weeks 0 , 1, and 2 [ $n=8$ for Ehmt $2^{f / f l}$ mice and $n=9$ for Ehmt2 $2^{f l / f l} \mathrm{Tg}($ Myh6-MCM) mice]. (F and G) qRT-PCR showing expression levels of Nppa, Nppb, Myh6, Myh7 (F), and collagen, type I, $\alpha 1$ (Col1a1) (G) in the LVs of Ehmt $2^{f / f f l}$ and Ehmt $2^{f / f l} \mathrm{Tg}($ Myh6-MCM) mice, 2 weeks after tamoxifen injection and after sham or AB operations. Error bars indicate the SEM. $n=5$ replicates $(\mathbf{F}$ and $\mathbf{G}) .{ }^{*} P<0.05,{ }^{* *} P<0.01$, and ${ }^{* * *} P<0.001$, by nested ANOVA (A and $\mathbf{B}$ ) and Student's $t$ test (C-C).

performed at the same time, however, and the effect was due to differences in the gradient across the stenosis imposed by the banding procedure. Importantly, the gradients were the same in animals within each cohort (A-366 experiment and EhmtKO experiment) (Supplemental Figure 5O). Consistent with the lack of an additive effect of EHMT loss of function on cardiac phenotypic parameters, the expression of molecular markers of pathological hypertrophy was not further augmented by genetic or pharmacological inactivation of EHMTs, in keeping with our in vitro findings (Figure 6, F and G, and Supplemental Figure $5 \mathrm{~L})$. This suggested that EHMT1/2 loss was integral to the mechanism underlying pathological hypertrophy.

miR-217 overexpression destabilizes Ehmt1/2 mRNAs. We next investigated how Ehmt1/2 expression was regulated during 
A

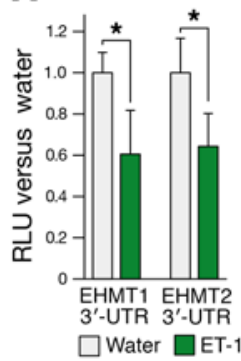

B

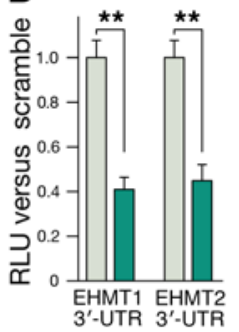

$\square$ Scramble $\square$ miR-217
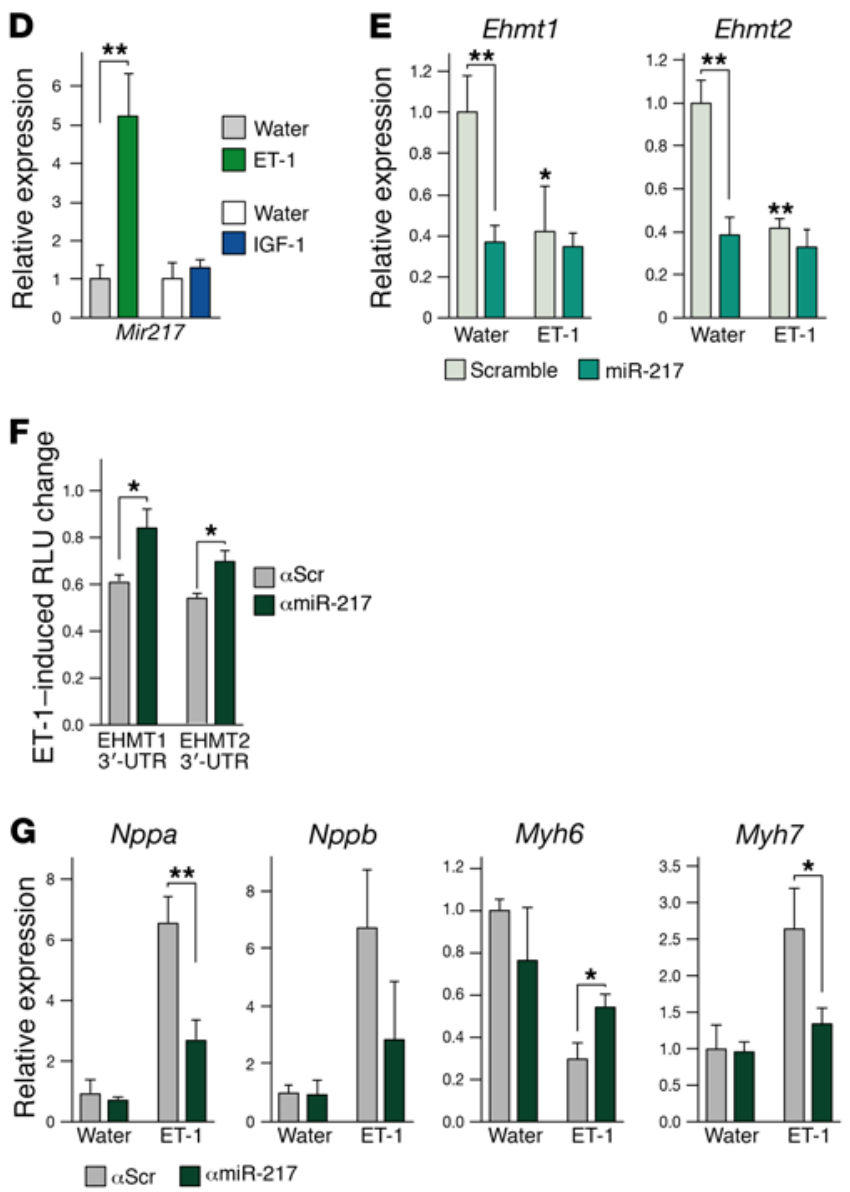

Figure 7. Downregulation of Ehmt1/2 by miR-217 is required for hypertrophy induction in vitro. (A and B) RLU of luciferase constructs harboring the 3'-UTR of EHMT1 or EHMT2, transfected into NRVMs treated with water or ET-1 (A), or into HEK293 cells transfected with scrambled or miR-217 mimic (B). (C and D) Expression of miR-217 in rat CM nuclei sorted from sham-operated, AB-operated, control, or exercised hearts (C), and in NRVMs treated for 48 hours with ET-1, IGF-1, or water (D). (E) Expression of Ehmt 1 and Ehmt2 in NRVMs transfected with scrambled or miR-217 mimic for 48 hours and treated for 48 hours with ET-1 or water. (F) RLU of luciferase constructs harboring the $3^{\prime}$-UTR of Ehmt1 or Ehmt2, transfected into NRVMs treated with control (anti-scramble; $\alpha \mathrm{Scr}$ ) or miR-217 antagomirs (anti-miR-217; $\alpha \mathrm{miR}-217$ ). Shown are the RLU in response to 48 hours of exposure to ET-1 relative to water-treated controls. (C) Expression of Nppa, Nppb, Myh6, and Myh7 in NRVMs treated with control or miR-217 antagomirs and exposed for 48 hours to water or ET-1. ${ }^{*} P<0.05$, ${ }^{* *} P<0.01$, and ${ }^{* * *} P<0.001$, by Student's $t$ test. Error bars represent the mean \pm SEM of 5 independent experiments. hypertrophy. Surprisingly, the observed loss of Ehmt1/2 mRNA expression was not evident from our nuclear expression analyses. Indeed, qRT-PCR confirmed that nascent Ehmt transcript levels were unaltered in CMs upon $\mathrm{AB}$, and likewise in NRVMs upon ET-1 treatment (Supplemental Figure 6, A and B). This suggested that pathological hypertrophy reduced Ehmt1/2 mRNA expression posttranscriptionally, possibly via a miR-dependent mechanism (33). To assess whether this effect was through mRNA destabilization via the 3'-UTR of Ehmt1/2, we generated luciferase reporter constructs harboring the $3^{\prime}$-UTRs of Ehmt1 and Ehmt2 and analyzed their activity in NRVMs treated with ET-1. Consistent with our hypothesis, ET-1 application reduced the luciferase activity of both reporters (Figure 7A). To identify the miR responsible for this effect, we scanned these 3 '-UTRs for miR-binding sites (seed sequences) using standard tools $(34,35)$. Of those identified, miR-217 was predicted to target both UTRs (Supplemental Figure 6C). We functionally confirmed this prediction, as transfection of a miR-217 mimic significantly reduced the activity of luciferase constructs containing either the Ehmt1 or Ehmt 2 3'-UTRs in HEK293 cells, whereas other predicted miRs were without consistent effects (Figure 7B and Supplemental Figure 6D). Supporting its role in hypertrophic remodeling and in line with an earlier report suggesting increased expression with $\mathrm{AB}$ (36), Mir217 expression was substantially augmented in vivo upon $A B$ but not exercise, and also in vitro in NRVMs upon treatment with ET-1 but not IGF-1 (Figure 7, C and D). The sufficiency of this increase in Mir217 for induction of hypertrophy was next tested. miR-217 mimic transfection reduced endogenous Ehmt levels in NRVMs and concomitantly triggered a pathological hypertrophic response (Figure 7E and Supplemental Figure 6E). Importantly, transfection of NRVMs with a miR-217 antagomir ( $\alpha$ miR-217) significantly blunted the ET-1-induced reduction in luciferase activity of both 3'-UTR reporters and of endogenous Ehmt1/2 mRNA levels (Figure 7F and Supplemental Figure 6F). Furthermore, miR-217 antagomir transfection attenuated the concomitant pathological hypertrophic response (Figure 7G). These effects of miR-217 were mediated by the direct regulation of Ehmt1/2 expression (and not through other targets of miR-217), as target site blockers that specifically prevent binding of miR-217 to their seed sequence in 3'-UTRs of Ehmt1/2 also blunted the ET-1-induced hypertrophic response (Supplemental Figure 6, G-J).

To examine the relevance of this pathway to hypertrophy in vivo, we suppressed miR-217 activity using locked nucleic acid-modified antagomirs and assessed the AB-induced hypertrophy. Mice subjected to $\mathrm{AB}$ or sham operation were injected with antagomirs targeting a scrambled miR or miR-217. This prevented the AB-induced reduction in EHMT1/2 expression and H3K9me2 levels (Figure 8A and Supplemental Figure 7, A and B). While no effects were noted in the sham-operated animals, the $\mathrm{AB}$ animals receiving miR-217 antagomirs showed markedly reduced $\mathrm{AB}$-induced pathological hypertrophy and its associated expression of fetal genes when compared with their scramble-injected counterparts (Figure 8, B-E), despite identical gradients over their banded aorta $(3.40 \pm 0.03 \mathrm{~m} / \mathrm{s}$ and $3.36 \pm 0.06 \mathrm{~m} / \mathrm{s}$ for scrambled and miR-217 antagomirs, respectively) (Supplemental Figure 7C). More specifically, the AB-induced LV weight increase was reduced by $46 \%$, the cardiac dimensions (concentricity) were better retained, and the decrease in cardiac function 
A
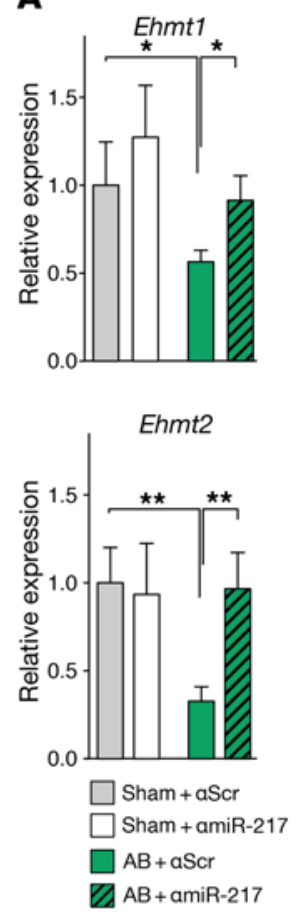

B

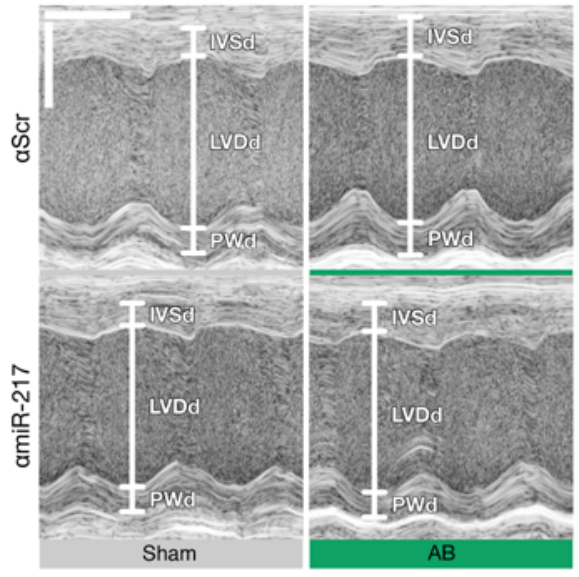

C

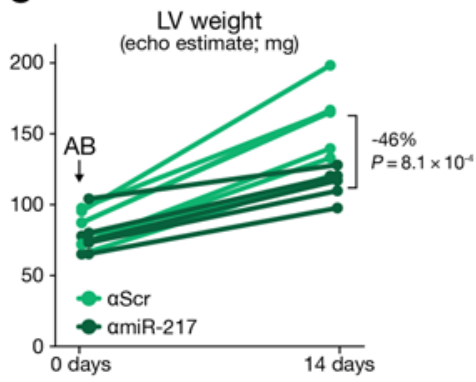

D
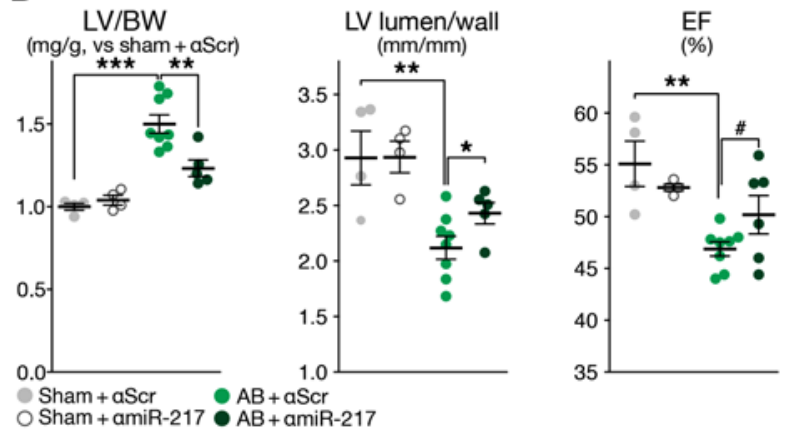

E
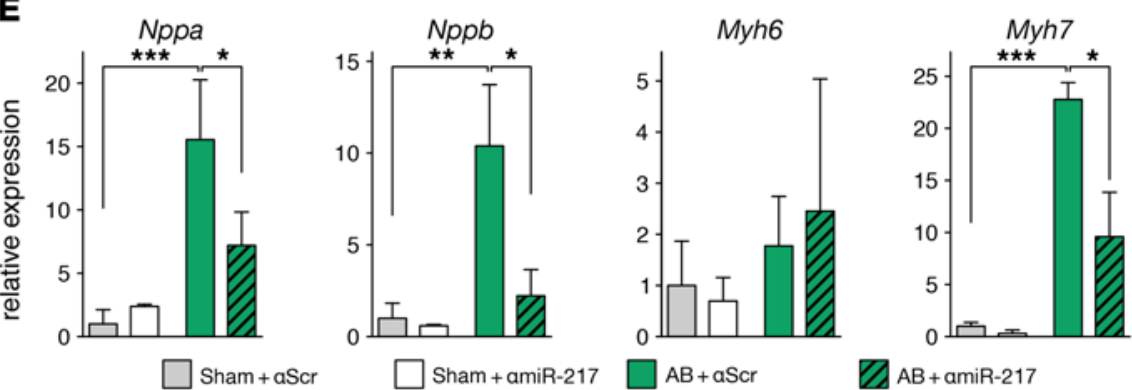

$\mathbf{F}$

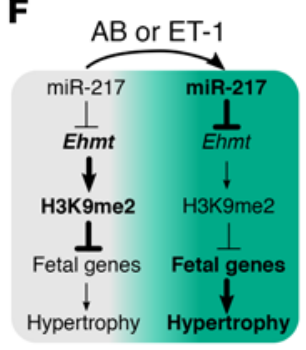

Figure 8. Downregulation of Ehmt1/2 by miR-217 is required for hypertrophy induction in vivo. (A) Effect of miR-217 antagomir or scramble treatment upon expression of Ehmt1 and Ehmt2 in hearts from sham- or AB-operated mice 2 weeks after surgery. (B) M-mode echocardiogram from parasternal location of mice treated as in A. Indicated are, at end-diastole, the LV diameter (LVDd) and the thickness of the posterior wall (PWd) and of the interventricular septum (IVSd). Horizontal scale: 0.1 second; vertical scale: $2 \mathrm{~mm}$. (C) LV mass as estimated by echocardiography (echo) 1 day after AB (before administration of antagomirs) and 2 weeks after $A B$ ( $n=5$ for miR-217- and 8 for scramble-injected animals). (D) LV/BW ratio, LV lumen/wall diameter ratio, and EF of hearts treated as in $\mathbf{A}(n=4,4,8,5$ for the indicated conditions). (E) Expression of Nppa, Nppb, Myh6, and Myh7 in hearts treated as in A. (F) Schematic of miR-217 regulation of Ehmt expression and pathological hypertrophy. ${ }^{* *} P<0.001,{ }^{* *} P<0.01,{ }^{*} P<0.05$, and ${ }^{*} P=0.07$, by Student's $t$ test. Error bars in $\mathbf{A}$ and $\mathbf{E}$ represent the mean \pm SEM of 4 (sham conditions), 8 (AB plus $\alpha$ Scramble), or 6 (AB plus $\alpha$ miR-217) mice.

(EF) was attenuated (Figure 8, B-D). Molecularly, this was mirrored by a decreased induction of the fetal heart gene marker panel (Figure 8E). This demonstrates that countering the Mir217 increase upon pathological hypertrophy is sufficient to attenuate the pathological hypertrophic response, not only in vitro but also in vivo. These findings indicate that increased expression of Mir217 upon induction of pathological hypertrophy reduces expression of Ehmt1/2 mRNAs to trigger a pathological hypertrophic response (Figure $8 \mathrm{~F}$ ).

Together, the data delineate a pathway in which miR-217associated loss of EHMT2 expression is sufficient to induce a pathological hypertrophic response characterized by cardiac phenotypic remodeling and reexpression of the fetal gene program. Notably, and consistent with our hypothesis, EHMT1/2 levels increased during the maturation of $\mathrm{CMs}$ from the fetal to adult stage, whereas Mir217 levels decreased, further indicating that this gene-regulatory axis is involved in CM maturation (Figure 9A). Our data therefore suggest a model in which the activity of EHMTs increases during $\mathrm{CM}$ maturation to suppress fetal gene expression programs and to protect the heart from the pathological hypertrophy associated with reexpression of this gene program (Figure 9B). 
A

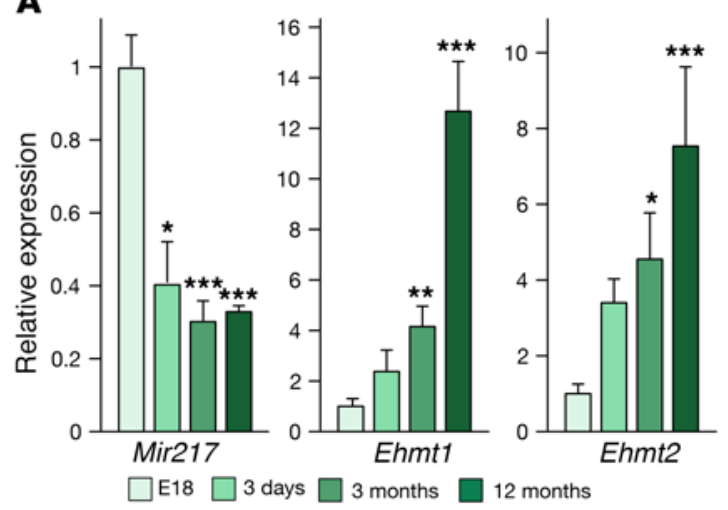

B

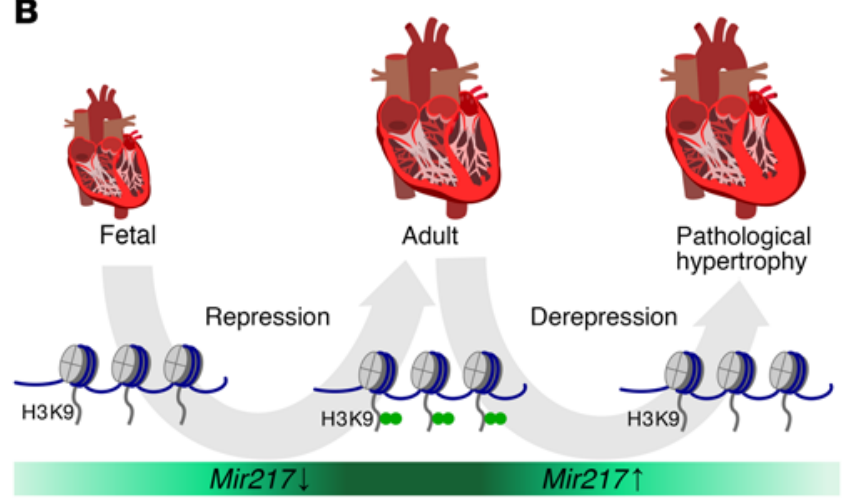

EHMT expression; H3K9me2

Fetal gene program (Nppa, Nppb, Myh7, ..)

Figure 9. Gain of Ehmt1/2 expression and loss of miR-217 during CM maturation. (A) qRT-PCR showing expression of Ehmt1, Ehmt2, and Mir217 in CMs isolated from rats at the indicated ages, relative to E18. Error bars represent the mean \pm SEM of 5 biological replicates. ${ }^{* *} P<0.001$, ${ }^{* *} P<0.01$, and ${ }^{*} P<0.05$, by Student's $t$ test. (B) Proposed role of EHMT-induced repression of the fetal gene program in suppressing pathological hypertrophic remodeling of the heart.

The miR-217/EHMT1/2/H3K9me2 axis is conserved in human cardiac hypertrophy. To assess the implications of these findings for cardiac hypertrophy in patients, we studied heart tissues obtained from autopsies of deceased males. $\mathrm{PCM}^{+}$nuclei were isolated by sorting from hypertrophied and normal hearts (Figure 10A and Supplemental Figure 8A). These purified CM nuclei displayed the anticipated changes in expression of fetal gene program markers of hypertrophy (Figure 10B). In line with our studies in rodents, nascent EHMT1/2 transcript levels were unaffected, whereas MIR217 expression showed a remarkable 5-fold increase in hypertrophied CMs (Figure 10, C and D). Elevated MIR217 expression was accompanied by a loss of EHMT1/2 mRNA expression and H3K9me2 levels in CMs (Figure 10, $\mathrm{E}$ and $\mathrm{F}$ ), a pathway that was also sufficient to reduce EHMT1/2 mRNA and protein expression as well as $\mathrm{H} 3 \mathrm{~K} 9 \mathrm{me} 2$ levels in a human cell line (Supplemental Figure 8, B-D). These data thus indicate a functional conservation of the identified miR-217/EHMT1/2/ H3K9me2 axis between rodents and humans.

\section{Discussion}

Hypertrophy induced by hypertension or aortic stenosis acts as a major risk factor for heart failure. Although physiological stressors also induce CM hypertrophy, the phenotype of the pathological response is substantially different, as it involves, among others, activation of the fetal gene program. What underlies this difference and how the adult cellular identity is bypassed to give rise to hypertrophic remodeling are largely unknown. Here, we identify what we believe to be a novel epigenetic-based mechanism that determines the different transcriptional responses of CMs during the development of physiological and pathological hypertrophy.

With the aim of identifying epigenetic changes that underlie phenotypic differences in the hypertrophic response, we profiled repressive histone marks, comparing, for the first time to our knowledge, hypertrophy induced by pathological and physiological stressors. Hypertrophy was of equivalent magnitude between both stimuli, and in the case of pathological hypertrophy was of a mild phenotype, since our analysis was aimed at gaining mechanistic insights into the development of hypertrophy. We did not study failing hearts, in which the pathological sequelae may be confounding. Performing our analysis on CM nuclei purified from in vivo hypertrophied hearts enabled us to directly record changes in the most relevant cell type and model. We observed that H3K9me2 was substantially and pervasively reduced upon pathological, but not physiological, hypertrophy of CMs in vivo. The reduction in H3K9me2 affected a large fraction of the chromatin ( $20 \%$ of all genes) and was also evident upon immunofluorescence and immunoblot analyses, underlining the magnitude of the loss. Reduced H3K9me2 affected regions where this mark was acquired during CM maturation, including those encoding fetal gene program markers ( $N p p a$, $N p p b$, and $M y h 7$ ) that are prototypically upregulated upon pathological hypertrophy. The affected genes were also more broadly involved in heart development, contraction, MAPK activity, and the response to hormonal stimuli - pathways similarly upregulated upon pathological hypertrophy. Expression levels of these genes were also increased during CM differentiation but downregulated in the postnatal heart, further asserting that they are characteristic of the fetal heart. Together, our data reinforce the notion that acquisition of H3K9 methylation is key to cells achieving their differentiated state. Although changes in epigenetic marks have been reported at specific loci and, in a few cases, genome wide $(37,38)$, we demonstrate a direct correlation in CMs between the epigenome and the transcriptome that underlies stimulus-specific pathological hypertrophy induction. Indeed, the overlaying of nascent RNA abundance and its changes during $\mathrm{AB}$ with alterations in the epigenome allowed us to determine an immediate correlation between the epigenome and active transcription at the time point analyzed, undiluted by posttranscriptional processes.

The enzymes responsible for depositing H3K9me2, EHMT1 and EHMT2, were downregulated through an increased expression of Mir217 upon pathological, but not physiological, hypertrophy. Although previously shown to be upregulated upon hypertrophy (36), the role of this miR in heart disease was otherwise unknown. miR-217-mediated, pharmacological, and genetic inhibition of EHMT1/2, moreover, demonstrated that the observed reduction in EHMT1/2 activity was not a mere epiphenomenon, but was sufficient to induce pathological hypertrophy in vitro in NRVMs and in vivo in mice. Prevention of the loss of EHMT1/2 during hypertrophy through suppression of miR-217 activity also counteracted the induction of hypertrophy. Although we do not exclude the possible 
A

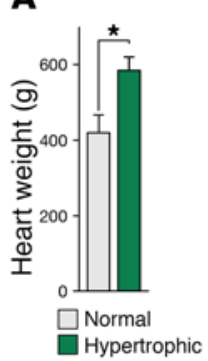

E

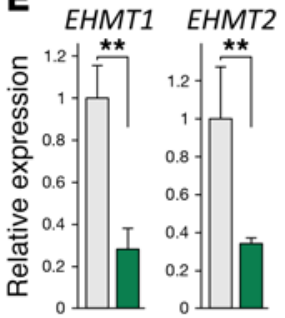

B

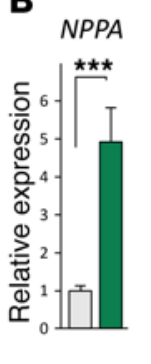

$\mathbf{F}$

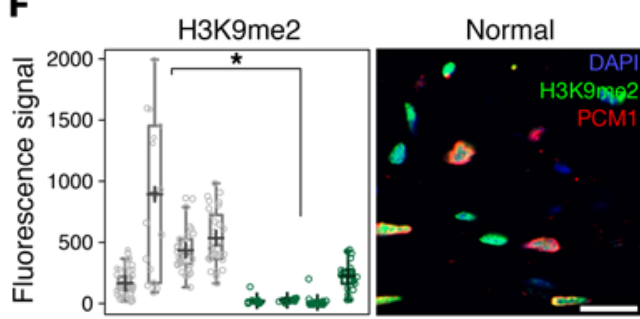

CIR217

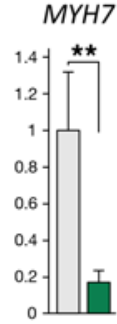

NPPB
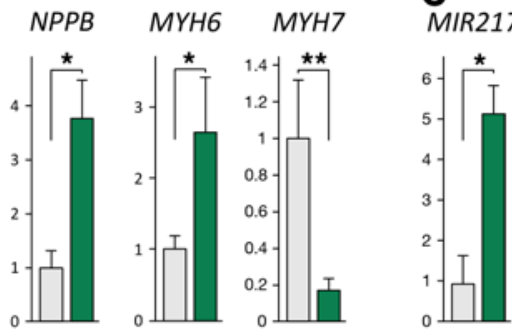

D
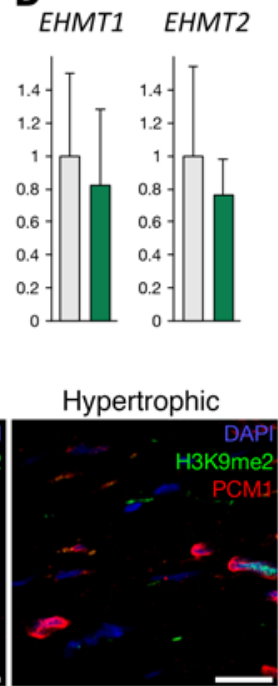

Figure 10. Loss of EHMT1/2 expression and H3K9me2 in human hypertrophic hearts. (A) Weight of normal and hypertrophic human hearts. (B-E) Expression of NPPA, NPPB, MYHG, and MYH7 (B), MIR217 (C), and EHMT1 and EHMT2 nascent transcripts (D) and mature mRNAs $(\mathbf{E})$ in $\mathrm{PCM} 1^{+}$nuclei in LVs of normal and hypertrophic human hearts. (F) Quantification of H3K9me2 signal in human CMs. Representative images of H3K9me2 staining (green) in $\mathrm{PCM}^{+} \mathrm{CM}$ nuclei (red) in normal and hypertrophic human hearts. Scale bars: $20 \mu \mathrm{m}$. Error bars represent the mean \pm SEM of $5(\mathbf{A}-\mathbf{E})$ and $4(\mathbf{F})$ biological replicates. ${ }^{*} P<0.05$, ${ }^{* *} P<0.01$, and ${ }^{* * *} P<0.001$, by Student's $t$ test $(\mathbf{A}-\mathbf{E})$ or nested ANOVA (F). existence of other routes leading to a loss of EHMT1/2 activity, our data demonstrate for the first time to our knowledge a potent role for miR-217 and the H3K9 dimethyltransferases EHMT1 and EHMT2 in the adult heart. EHMTs are essential for normal development, and mice with $\mathrm{KO}$ of either gene die at E9.5 due to severe growth defects $(20,39)$. In humans, heterozygous loss of EHMT1 likewise causes multiple developmental anomalies including cardiac defects (40), and we demonstrate here that EHMT1 and EHMT2 expression levels are downregulated upon pathological hypertrophy. Our results contrast with those of a recent study reporting a modest increase in Ehmt2 during hypertrophy and alterations of H3K9me2 at Myh6 (41). A direct comparison of this study with our data is, however, challenging, given the lack of CM-specific analysis, the restriction of analysis solely to the Myh6 locus, as well as the differences in animal models analyzed in the other study. EHMT2 is essential for appropriate differentiation of a wide range of cell types including neurons, adipocytes, skeletal myocytes, Th2 cells, and photoreceptors (12, 42-45). A recent study similarly implicated EHMT1/2 in murine prenatal heart development, although precise cellular phenotypes were not described (46). Interestingly, in terminally differentiated neurons, acute loss of EHMTs derepresses the expression of a number of neuronal progenitor genes (12), indicating that the H3K9me2 mark is necessary not only for the establishment but also for the maintenance of a terminally differentiated neuronal phenotype. Importantly, EHMT1/2 inhibition in adult neurons as well as in brown adipose tissue leads to an ectopic, heterochronic expression of fetal CM-specific genes such as $M y h 7(12,13,47)$. Our current data indicate that EHMTs also repress these fetal CM-specific genes paratopically in the adult $\mathrm{CM}$.

Altogether, our findings suggest a model wherein H3K9me2 accumulates during development and keeps the adult CM locked in its terminally differentiated state, suppressing the fetal gene program. Through pathological hypertrophy-driven expression of miR-217 and loss of EHMT1/2, concomitantly with a reduction in $\mathrm{H} 3 \mathrm{~K} 9 \mathrm{me} 2$, the CM genome is derepressed, allowing progression to a less differentiated state and providing the basis for a widespread, pathological remodeling of the CM transcriptome. The loss of H3K9 dimethylation that occurs upon pathological, but not phys- iological, CM hypertrophy thus highlights that enzymes involved in $\mathrm{H} 3 \mathrm{~K} 9 \mathrm{me} 2$ metabolism represent appealing targets for the treatment of heart disease, either directly or by inhibiting miR-217.

\section{Methods}

$A B$. Male Sprague Dawley rats ( $170 \mathrm{~g}$; Taconic) and C57BL/6J mice (15-18 g; Janvier and Taconic) were anesthetized, and a 3-0 (rats) or 8-0 (mice) silk suture was tightened around the ascending aorta proximal to the brachiocephalic trunk. Sham-operated animals served as controls. For rats, only those having nonfailing hearts with detectable hypertrophy after 6 weeks were retained. For mice, those with a mild stenosis (2.5-3.5 m/s in maximal flow velocity over aortic stenosis measured on the first or second postoperative day as indicated in the Supplemental Methods) and no signs of congestion (left anterior descending artery [LAD] $<2.0 \mathrm{~mm}$ ) were included.

Exercise training. Male Sprague Dawley rats (Taconic) Jweighing approximately $280 \mathrm{~g}$ were assigned to interval training on a treadmill (Columbus Instruments) at a 25-degree inclination, with 5 days of acclimatization at a running velocity of $6 \mathrm{~m} / \mathrm{min}$ for $30,45,60,75,90$, and 120 minutes. Thereafter, interval training was performed 6 days a week in twelve 8-minute intervals, with a 2 -minute resting period (6 $\mathrm{m} / \mathrm{min}$ ) after a warm-up period at $10 \mathrm{~m} / \mathrm{min}$. Exercise training was performed over a 6-week period at a running speed of $15 \mathrm{~m} / \mathrm{min}$ that was increased by $2 \mathrm{~m} / \mathrm{min}$ each week.

A-366 treatment, CM-specific Ehmt2 KO, and antagomir delivery. On the second postoperative day, mice were stratified by stenosis and randomized to a 10-day treatment with either $30 \mathrm{mg} / \mathrm{kg} / \mathrm{d}$ A-366 dissolved in 98:2 PEG 400/polysorbate 80 or control treatment with 98:2 PEG 400/polysorbate 80 without active compound, administrated s.c. through miniosmotic pumps. Ehmt $2^{f / f l}$ mice (48) were crossed with CM-restricted (Myh6 promoter), tamoxifen-inducible MerCreMer-expressing transgenic mice (47) to generate C57BL/6J $E h m t 2^{f / f l} \operatorname{Tg}(M y h 6-\mathrm{MCM})$ mice. Littermate $E h m t 2^{f / f l}$ mice were used as controls unless otherwise specified. Tamoxifen $(40 \mathrm{mg} / \mathrm{kg})$ was administrated on the first postoperative day by a single i.p. injection. To test the in vivo effect of miR-217 upregulation, 7-week-old C57BL/6J mice were randomized on the first postoperative day to miR-217 or scrambled antagomir treatment, on echocardiographic 
examination. Locked nucleic acid oligonucleotides (Exiqon) targeting miR-217 (AGTTCCTGATGCAGT) or a scrambled control (ACGTCTATACGCCCA) were dissolved in PBS (4 mg/ml), and $10 \mathrm{mg} / \mathrm{kg}$ was injected into the jugular vein 1 and 4 days after surgery. Magnetic resonance imaging (MRI) and echocardiography were done 14 days after surgery, before hearts were harvested.

Human heart tissues. Heart specimens were provided by the Department of Forensic Medicine in Stockholm, Sweden. The myocardial LVs were collected after informed consent from relatives was received and were stored at $-80^{\circ} \mathrm{C}$ until further use.

In vitro models of hypertrophy. Ventricular CMs from neonatal (P3) Sprague Dawley rats (NRVMs) (obtained from Harlan and Charles River Laboratories) were isolated and cultured as described previously (49). NRVMs were serum starved for 24 hours and left either unstimulated or stimulated for 48 hours with $100 \mathrm{nM} \mathrm{ET-1}$ or $10 \mathrm{nM} \mathrm{IGF-1} \mathrm{(both} \mathrm{from}$ EMD Millipore) in the presence of UNC0638 (350 nM), A-366 (0.5 $\mu \mathrm{M})$, or its carrier (DMSO). miR studies were performed as previously described (49), using compounds specified in the Supplemental Methods. EHMT2 was overexpressed through adenoviral transduction, with empty virus serving as a control (26). After 8 hours, cells were treated with ET- 1 and incubated for an additional 48 hours prior to harvesting.

Nuclear isolation and sorting. Nuclei were isolated and sorted from 2 LV wall samples per experiment: 1 hypertrophic sample and 1 corresponding control sample. Nuclear isolation and sorting were based on a previously described method (5), as detailed in the Supplemental Methods.

ChIP-seq and RNA-seq. ChIP-seq and strand-specific RNA-seq were performed according to standard methods using in-house protocols based on NEBnext kits (New England BioLabs Inc.). Forty-six bases of these libraries were sequenced on an Illumina HiSeq 1000 and aligned using Bowtie against the rat genome build 3.4. Circular binary segmentation identified LOCKs and BLOCs, and differences in histone methylation herein were required to differ by at least $20 \%$ and to have an FDR-corrected $P$ value below 0.1. Additional details are provided in the Supplemental Methods. Data are available in the NCBI's Gene Expression Omnibus (GEO) database (GEO GSE66653).

Immunofluorescence analyses. Immunofluorescence analyses were performed by standard methods, as detailed in the Supplemental Methods and in ref. 49.

Data representation and statistical analysis. Comparisons were made using a homoscedastic 2-tailed Student's $t$ test or ANOVA with a posthoc Tukey's honest significant difference (HSD) test when comparing, respectively, 2 or more than 2 conditions. Nested ANOVA was applied to immunofluorescence data sets, as multiple measurements per sample were available. A $P$ value of less than 0.05 was considered significant.

Study approval. Experiments involving animals were performed in accordance with United Kingdom Home Office and European Union guidelines and were approved by the Animal Welfare and
Ethical Review Body of the Babraham Institute (United Kingdom) and conformed to the Regulations on Animal Experimentation under The Norwegian Animal Welfare Act, approved by the Norwegian Animal Research Authority (FDU applications 3301, 3820, and 5338). Permission for the analysis of human tissue was granted by the Regional Ethics Committee in Stockholm, Sweden.

\section{Author contributions}

Study conceptualization: BT, WR, and HLR. Methodology: BT, HLR, JMA, and OB. Software, visualization, and formal analysis: BT, HO, ELR, HLR, and JMA. Validation and investigation: BT, ELR, HLR, JMA, HO, EL, AF, PB, KA, AT, and AA. Resources: JMA, IS, PB, and KA. Data curation: BT, JMA, and ELR. Writing of the original draft: BT and HLR. Writing, review, and editing of the manuscript: WR, JMA, ELR, and IS. Supervision: IS, WR, and HLR. Project administration: HLR. Funding acquisition: BT, WR, IS, and HLR.

\section{Acknowledgments}

BT was supported by a Marie Curie Intra-European Fellowship (SEARCH, 253644) and a Babraham Institute Synergy grant (to HLR and WR); EL and AF were supported by Leonardo Da Vinci Unipharma - Graduates studentships; AA was supported by a Cambridge Trust Scholarship; and ELR was supported by a studentship from the Wellcome Trust. Work in the HLR laboratory was funded by the Babraham Institute, The Royal Society, an Institute Strategic Programme Grant from the Biotechnology and Biological Sciences Research Council (BBSRC), and an Odysseus Award from the Research Foundation Flanders FWO (Fonds Wetenschappelijk Onderzoek). HLR would also like to acknowledge Prof. K. Sipido and the Department of Cardiovascular Sciences at KU Leuven for assistance in establishing his laboratory. Work in the OB laboratory was funded by the Karolinska Institutet, the Swedish Research Council, the Ragnar Söderberg Foundation, the Jeansson Foundations, and the Åke Wibergs Foundation. JMA and IS were supported by the KG Jebsen Cardiac Research Center and the Center for Heart Failure Research of the University of Oslo and by the Anders Jahres Fund for the Promotion of Science. The Oslo Preclinical MR Core Facility, represented by Lili Zhang and Vidar Skulberg, provided technical assistance. We thank Peter Brown and the Structural Genomics Consortium (Toronto, Canada) for the gift of A-366 and Makoto Tachibana (Kyoto University, Kyoto, Japan) for the $E h m t 2^{f / f l}$ mice.

Address correspondence to: Llewelyn Roderick, CDG 9th Floor, Herestraat 49, B3000 Leuven, Belgium. Phone: 3216377150; E-mail: llewelyn.roderick@kuleuven.be. Or to: Bernard Thienpont, Herestraat 49, O\&N4 Box 912, B3000 Leuven, Belgium. Phone: 3216373208; E-mail: bernard.thienpont@vib-kuleuven.be.
1. Mathers CD, Loncar D. Projections of global mortality and burden of disease from 2002 to 2030. PLoS Med. 2006;3(11):e442.

2. Heineke J, Molkentin JD. Regulation of cardiac hypertrophy by intracellular signalling pathways. Nat Rev Mol Cell Biol. 2006;7(8):589-600.

3. Soonpaa MH, Field LJ. Survey of studies examining mammalian cardiomyocyte DNA synthesis.
Circ Res. 1998;83(1):15-26.

4. Bergmann O, et al. Dynamics of Cell Generation and Turnover in the Human Heart. Cell. 2015;161(7):1566-1575.

5. Bergmann O, et al. Evidence for cardiomyocyte renewal in humans. Science. 2009;324(5923):98-102.

6. Olson EN, Schneider MD. Sizing up the heart: development redux in disease. Genes Dev. 2003;17(16):1937-1956.

7. Allard MF, Schönekess BO, Henning SL, English DR, Lopaschuk GD. Contribution of oxidative metabolism and glycolysis to ATP production in hypertrophied hearts. Am J Physiol. 1994;267(2 Pt 2):H742-H75O

8. Miyata S, Minobe W, Bristow MR, Leinwand LA. 
Myosin heavy chain isoform expression in the failing and nonfailing human heart. Circ Res. 2000;86(4):386-390.

9. Delgado-Olguín $\mathrm{P}$, et al. Epigenetic repression of cardiac progenitor gene expression by Ezh2 is required for postnatal cardiac homeostasis. Nat Genet. 2012;44(3):343-347.

10. Haberland M, Montgomery RL, Olson EN. The many roles of histone deacetylases in development and physiology: implications for disease and therapy. Nat Rev Genet. 2009;10(1):32-42.

11. Nührenberg TG, et al. Cardiac myocyte de novo DNA methyltransferases $3 \mathrm{a} / 3 \mathrm{~b}$ are dispensable for cardiac function and remodeling after chronic pressure overload in Mice. PLoS One. 2015;10(6):e0131019.

12. Schaefer A, et al. Control of cognition and adaptive behavior by the GLP/G9a epigenetic suppressor complex. Neuron. 2009;64(5):678-691.

13. Ohno H, Shinoda K, Ohyama K, Sharp LZ, Kajimura S. EHMT1 controls brown adipose cell fate and thermogenesis through the PRDM16 complex. Nature. 2013;504(7478):163-167.

14. Gonzalez-Sandoval A, et al. Perinuclear anchoring of H3K9-methylated chromatin stabilizes induced cell fate in C. elegans embryos. Cell. 2015;163(6):1333-1347.

15. Wen B, Wu H, Shinkai Y, Irizarry RA, Feinberg AP. Large histone $\mathrm{H} 3$ lysine 9 dimethylated chromatin blocks distinguish differentiated from embryonic stem cells. Nat Genet. 2009;41(2):246-250.

16. Pauler FM, et al. H3K27me3 forms BLOCs over silent genes and intergenic regions and specifies a histone banding pattern on a mouse autosomal chromosome. Genome Res. 2009;19(2):221-233.

17. Filion GJ, et al. Systematic protein location mapping reveals five principal chromatin types in Drosophila cells. Cell. 2010;143(2):212-224.

18. Wamstad JA, et al. Dynamic and coordinated epigenetic regulation of developmental transitions in the cardiac lineage. Cell. 2012;151(1):206-220.

19. O'Meara CC, et al. Transcriptional reversion of cardiac myocyte fate during mammalian cardiac regeneration. Circ Res. 2015;116(5):804-815.

20. Tachibana M, et al. Histone methyltransferases G9a and GLP form heteromeric complexes and are both crucial for methylation of euchromatin at H3-K9. Genes Dev. 2005;19(7):815-826.

21. Shinkai Y, Tachibana M. H3K9 methyltransferase G9a and the related molecule GLP. Genes Dev.
2011;25(8):781-788.

22. Yokochi T, et al. G9a selectively represses a class of late-replicating genes at the nuclear periphery. Proc Natl Acad Sci U S A. 2009;106(46):19363-19368.

23. Guelen L, et al. Domain organization of human chromosomes revealed by mapping of nuclear lamina interactions. Nature. 2008;453(7197):948-951.

24. Whetstine JR, et al. Reversal of histone lysine trimethylation by the JMJD2 family of histone demethylases. Cell. 2006;125(3):467-481.

25. Greer EL, Shi Y. Histone methylation: a dynamic mark in health, disease and inheritance. Nat Rev Genet. 2012;13(5):343-357.

26. Higazi DR, et al. Endothelin-1-stimulated InsP3-induced $\mathrm{Ca} 2+$ release is a nexus for hypertrophic signaling in cardiac myocytes. Mol Cell. 2009;33(4):472-482.

27. Shubeita HE, et al. Endothelin induction of inositol phospholipid hydrolysis, sarcomere assembly, and cardiac gene expression in ventricular myocytes. A paracrine mechanism for myocardial cell hypertrophy. J Biol Chem. 1990;265(33):20555-20562.

28. Molkentin JD, Dorn GW. Cytoplasmic signaling pathways that regulate cardiac hypertrophy. Anпи Rev Physiol. 2001;63:391-426.

29 . Vedadi M, et al. A chemical probe selectively inhibits G9a and GLP methyltransferase activity in cells. Nat Chem Biol. 2011;7(8):566-574.

30. Sweis RF, et al. Discovery and development of potent and selective inhibitors of histone methyltransferase g9a. ACS Med Chem Lett. 2014;5(2):205-209.

31. Bernardo BC, Weeks KL, Pretorius L, McMullen JR. Molecular distinction between physiological and pathological cardiac hypertrophy: experimental findings and therapeutic strategies. Pharmacol Ther. 2010;128(1):191-227.

32. Nakayama $\mathrm{H}$, et al. The IP3 receptor regulates cardiac hypertrophy in response to select stimuli. Circ Res. 2010;107(5):659-666.

33. Condorelli G, Latronico MV, Dorn GW. microRNAs in heart disease: putative novel therapeutic targets? Eur Heart J. 2010;31(6):649-658

34. John B, Enright AJ, Aravin A, Tuschl T, Sander C, Marks DS. Human MicroRNA targets. PLoS Biol. 2004;2(11):e363.

35. Krek A, et al. Combinatorial microRNA target predictions. Nat Genet. 2005;37(5):495-500.

36. van Rooij E, et al. A signature pattern of stress-re- sponsive microRNAs that can evoke cardiac hypertrophy and heart failure. Proc Natl Acad Sci US A. 2006;103(48):18255-18260.

37. Gilsbach R, et al. Dynamic DNA methylation orchestrates cardiomyocyte development, maturation and disease. Nat Commun. 2014;5:5288.

38. Hohl M, et al. HDAC4 controls histone methylation in response to elevated cardiac load. JClin Invest. 2013;123(3):1359-1370.

39. Tachibana M, et al. G9a histone methyltransferase plays a dominant role in euchromatic histone $\mathrm{H} 3$ lysine 9 methylation and is essential for early embryogenesis. Genes Dev. 2002;16(14):1779-1791.

40. Kleefstra T, et al. Loss-of-function mutations in euchromatin histone methyl transferase 1 (EHMT1) cause the 9q34 subtelomeric deletion syndrome. Am J Hum Genet. 2006;79(2):370-377.

41. Han P, et al. Epigenetic response to environmental stress: Assembly of BRG1-G9a/GLP-DNMT3 repressive chromatin complex on Myh6 promoter in pathologically stressed hearts. Biochim Biophys Acta. 2016;1863(7 Pt B):1772-1781.

42. Wang L, et al. Histone H3K9 methyltransferase G9a represses PPAR $\gamma$ expression and adipogenesis. EMBO J. 2013;32(1):45-59.

43. Ling BM, et al. Lysine methyltransferase G9a methylates the transcription factor MyoD and regulates skeletal muscle differentiation. Proc Natl Acad Sci U S A. 2012;109(3):841-846.

44. Lehnertz B, et al. Activating and inhibitory functions for the histone lysine methyltransferase G9a in Thelper cell differentiation and function. JExp Med. 2010;207(5):915-922.

45. Thomas LR, et al. Functional analysis of histone methyltransferase g9a in B and T lymphocytes. JImmunol. 2008;181(1):485-493.

46. Inagawa M, et al. Histone $\mathrm{H} 3$ lysine 9 methyltransferases, G9a and GLP are essential for cardiac morphogenesis. Mech Dev. 2013;130(11-12):519-531.

47. Sohal DS, et al. Temporally regulated and tissue-specific gene manipulations in the adult and embryonic heart using a tamoxifen-inducible Cre protein. Circ Res. 2001;89(1):20-25.

48. Tachibana M, Nozaki M, Takeda N, Shinkai Y. Functional dynamics of $\mathrm{H} 3 \mathrm{~K} 9$ methylation during meiotic prophase progression. $E M B O J$. 2007;26(14):3346-3359.

49. Drawnel FM, et al. Mutual antagonism between IP(3)RII and miRNA-133a regulates calcium signals and cardiac hypertrophy. J Cell Biol. 2012;199(5):783-798 